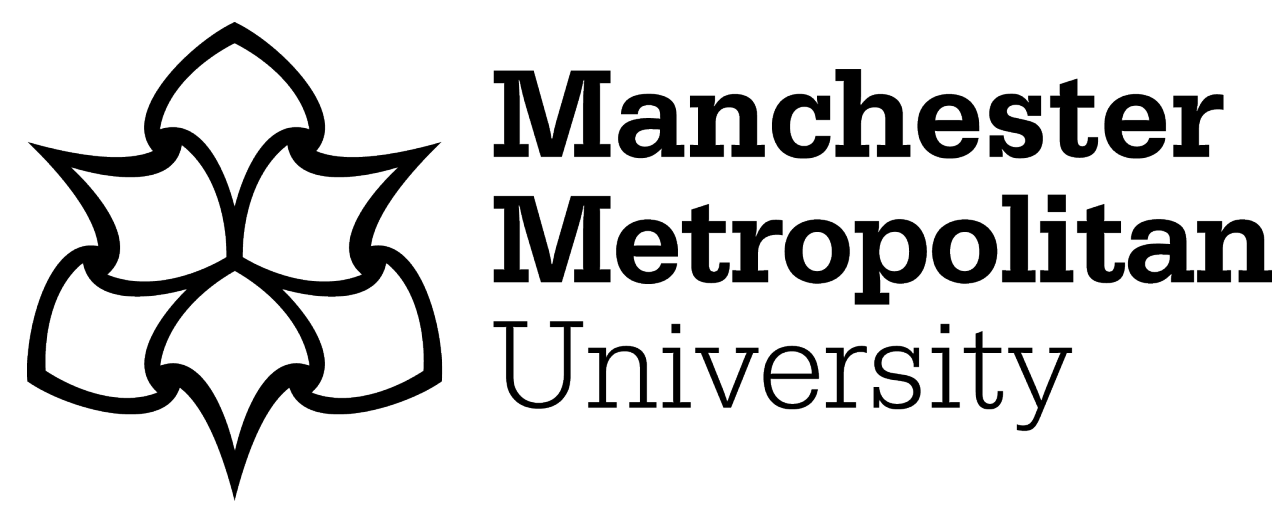

Abbasi, R, Kashif Bashir, A, Jianwen, C, Mateen, A, Piran, J, Amin, F and Luo, B (2021) Author classification using transfer learning and predicting stars in co-author networks. Software - Practice and Experience, 51 (3). pp. 645-669. ISSN 0038-0644

Downloaded from: https://e-space.mmu.ac.uk/626670/

Version: Accepted Version

Publisher: Wiley

DOI: https://doi.org/10.1002/spe.2884

Please cite the published version 


\title{
Author Classification using Transfer Learning and Predicting Stars in Co-Author Networks
}

\author{
Rashid Abbasi $^{1}$ | Ali Kashif Bashir ${ }^{2}$ | Abdul Mateen ${ }^{3}$ | Farhan Amin ${ }^{4}$ | Bin Luo ${ }^{1}$ Chen Jian \\ $\mathrm{Wen}^{5}$
}

\footnotetext{
${ }^{1}$ Key Lab of Intelligent Computing and Signal Processing of MOE \&, School of Computer and Technology,Anhui University, Hefei, P.R.China

2 Department of Computing and Mathematics, Manchester

Metropolitan University, Manchester, United Kingdom

${ }^{3}$ Department of Computer Science, Federal Urdu University of Arts Science and Technology, Islamabad, Pakistan.

${ }^{4}$ Department of Information and Communication engineering, Yeungnam University, Yeungnam, South Korea

${ }^{5}$ Department of Computer and Technology, University of Electronics Science and Technology of China, Chengdu, Sichuan,, China

\section{Correspondence \\ *Corresponding Rashid Abbasi Email: rashidd.abbasi@gmail.com}

Present Address

This is sample for present address text this is sample for present address text

\begin{abstract}
The vast amount of data is key challenge to mine a new scholar that is plausible to be star in the upcoming period. The enormous amount of unstructured data raise every year is infeasible for traditional learning; consequently, we need a high quality of preprocessing technique to expand the performance of traditional learning. We have persuaded a novel approach, Authors classification algorithm using Transfer Learning $(A C T L)$ to learn new task on target area to mine the external knowledge from the source domain. Comprehensive experimental outcomes on real-world networks showed that (ACTL), Nodebased Influence Predicting Stars (NICPS), Corresponding Authors Mutual Influence based on Predicting Stars (CAMIPS) and Specific Topic Domainbased Predicting Stars (STDPS) enhanced the node classification accuracy as well as predicting rising stars to compared with contemporary baseline methods.
\end{abstract}

\section{KEYWORDS:}

Social network; Transfer learning; Prediction; Author classification

\section{1 | INTRODUCTION}

Academic Social Networks ( $A S N$ ) based on co-author and citation oriented relationships between authors, research articles, and co-authors contribution in different research article ${ }^{1}$. $A S N$ task as a professional finding ${ }^{2}$, professional interest finding ${ }^{3}$, tag disambiguation ${ }^{2145}$, citation recommendations ${ }^{6}$, and rising star finding. This work reveals the predicting rising stars to predict new researchers with skills to become an expert in future while the scholars with a low profile but having a potential to be on top in future are referred as rising stars ${ }^{7}$. Previously, when a new researcher was contributing with a senior researcher on the high-rank publication venue had more chances to be a star in the future. $A S N^{[89}$ based on co-authorship, co-reference relationship and social tagging 10 . Microsoft Academic Search and Arnetminer are online services which store the information of scholars such as predicting experts ${ }^{11}$ and research collaboration $12|13| 14 \mid 15$. 
Li et al ${ }^{17}$ focused the mutual influence and static score of venues; moreover, author ${ }^{16}$ suggested the dynamic author's research profiles by grouping the authors inversely by using non-supervised learning approaches. PubRank ${ }^{7}$ algorithm upgraded by StarRank ${ }^{11}$ method which considered the author contributions based on mutual influence. The objective is to find the rising stars which result "Weather a scholar to be rising star or not in future"13114. PageRank 17 is a key feature finding technique from the graph, extracting the keywords, and key phrases from documents which further attained by TextRank $\frac{18}{18}$. PubRank ${ }^{77}$ proposed for academic social networks for predicting the rising stars to consider the mutual influence and previous record of scholar publications on different venue; moreover, StarRank ${ }^{1}$ based on PubRank to magnify the co-author contribution and mutual influence from researchers networks. Rising stars prediction which is a small work has been carried out in academic social networks while the main idea is that whether a junior researcher work with expert researcher having chances to be expert in future.

\section{1 | Key Challenges and Motivations}

The key challenge is to mine the future predicting stars from two or more different networks who will be a predicting star in forthcoming. Normally the social networks are designed for a particular purpose and every network have its own entities representing by its nodes and related links which shows the different relationship between the nodes. Apart from the different network's relationship, for example: in a citation relationship of two diverse networks both have their own features for nodes. it is unable to get accurate classification through traditional machine learning. The most significant to compute the influence of one researcher to other researchers as well as the first researcher is generally pondered to be a top contributor as compare with second and so on. Moreover, PubRank method ${ }^{7}$ used the non-dynamic ranking method which does not deliver the newest ranking list of publication venues. StarRank ${ }^{1}$ calculated the mutual influence of author contribution which does not consider the corresponding author weight, besides didn't determine the track record of author research area with respect of author publication venues.

The massive amount of unstructured data is increasing on a different domain on the web is a key challenge due to infeasible of traditional learning as a result, we need a high quality of classification approach to expand the performance of machine learning techniques to predict future rising stars 192012122.

Two networks are frequently exchanged some similar features; however, the information of nodes features is not exchangeable. For example, two citation networks, the CiteSeer network having 3,327 nodes, while 2,708 nodes in Cora network. Both networks have no similarities in nodes or edge; however, both the networks exchange some common sub-graph that represents some frequent structures patterns with prominent similarities in the cross-network. It's very problematic to classify unlabeled nodes to train a virtuous classifier due to the deficiency of labeled node; moreover, mutually classification can give a higher classification consequence compare with traditional-leaning techniques ${ }^{23124}$. A learning framework required well-connected information to achieve the learning objective for an authorąís classification. In cross authors networks, a node exchange its common feature to achieve the accurate performance for authors classification; therefore, it is required to have the same structure in networks and generalize sub-graph for transferring the related structural features which frequently occur between two networks to improve the author classification in target network.

\subsection{Contribution}

The aim of transfer learning is to learn a new task in the target area to mine external knowledge from source area ${ }^{25}$. We proposed a new method for author classification via cross-network transfer learning. Our main idea is to explore the similar signature graphs patterns between the networks i.e. source network and the target network, which bring the improvement in author classification of the target network. (1) In the first phase, we have used state of the art method for training and validation from the source domain, Author based Classification algorithm using Transfer Learning (ACTL).

(2) In the second phase, there are different kinds of challenges in social influences analysis. The first challenge is that how to control the network structure to computes the social influence. The influence between each other does not only depend upon their own topics but also have a social relationship with other authors. The main task is to find a unique approach to utilize both the local attributes i.e. topic distribution and the global structure of network information for the analysis of social influence. How to compute social influence score, we can discriminate with 
diverse angles, and how to discover the strength of social influence with respect of fair contribution within a specific domain. Consequently, we need to quantify the mutual influence score to predict the precise future rising stars.

We proposed a new algorithm, Corresponding Author Mutual Influence (CAMI), Node base Influence Score (NIC) to compute mutual influence score auxiliary, predict expert finding using NIC based Predicting Stars (NICPS), CAMI based Predicting Stars finding (CAMIPS), Specific Topic domain based Predicting Stars finding (STDPS).

Prearranged our remainder part as follows. Section 1, described the introduction. Section 2, we have discussed related work, and section 3, described the proposed algorithms. Section 3.1, and subsection defined different nodeweighting schemes, section 3.2, and subsections defined the predicting stars finding by using different schemes. Performance evaluation result described in section 4 and the conclusion finalized in Section 5.

\section{2 | RELATED WORK}

In the field of social network and data mining, one of the important learning tasks is the author classification from different networks. For instance, we have a number of nodes and some of them are labeled while some are unlabeled. The combination of structural features and content of the labeled nodes to classify the unlabeled node 2627 . The collective classification achieves higher classification accuracy compared with the individual classification methods shown in the previous techniques $28 \mid 29$. A collective classification method to decrease the learning and inference changes within the domains whereas the same set of nodes are connected by multiple networks ${ }^{30}$. Transfer learning is efficaciously useful in many application area of machine learning like, image classification ${ }^{31}$, text classification ${ }^{27}$, and human activity classification $28|29| 30$.

\section{$2.1 \quad$ Transfer Learning}

The data mining and machine learning have many applications where data is extracted from one domain to visualize into other domain. However, in traditional learning, the source domain (training data) and the target domain (testing data) have the same data distribution and feature space beside in case of difference in source and target domain the predictive learner result can be decline to predict the rising stars 32114 . The main inspiration of transfer learning is to improve the performance of learners to the optimum level of target data and get the information from the relevant domain where the labeled data is very small in numbers ${ }^{33}$. Traditional machine learning result is a decline for the reason of the difference in domain data while Transfer leaning, the core intention is to apply knowledge and extract the key information from other domain.

In the first class, instance transfer method is used to transfer an information $\frac{3435}{35}$ in which the instance weighting in the target domain, the most parts of instances are reused for learning and assign larger weights in both the source and target domain 34 . The common parameter method is lying in the second category of transferring the information where the source and target learning task share the same parameter 36 [37/38. The lack of a labeled node is the main concern for the classifier to predict the unlabeled node. In many social networks, the content feature is not allied with the node; however, they are the mutual influence and share common dependency 23143144 . Mutually classification can give higher classification outcome to compare with traditional-leaning method ${ }^{45 / 46}$. Mutual social influence is an important factor to compute the influence between two nodes. The graphical model generally we can use to predict the social connections for training and prediction of the corresponding relationship attributes to increase the labels of conditional probability. Many graphical models are extensively used to designate the dependencies between the data like Restricted Boltzmann Machine ${ }^{47}$ factor graph $\underline{48}$.

The frequent sub-graph mining 49150 and influence pattern finding in a large network to transfer those sub-graph structures whose threshold is above the minimum support in the graph data-set 51 . The high-frequency pattern plays a very important role in graph database indexing 46 . For finding the recurrent subgraph pattern, an adjacency matrix is used to present the graph in AGM method 49 . GSpan ${ }^{50}$ method to avoid the graph isomorphism in which the frequently connected subgraph has effectively adopted a new lexicographic order to map each graph by using depthfirst search (DFS). We proposed to find a similar subgraph from both the networks i.e. source and target networks to make it possible to transfer the information between two networks to compute the author influence/ author weight and future predicting stars. 


\section{3 | AUTHOR BASED CLASSIFICATION ALGORITHM USING TRANSFER LEARNING (ACTL)}

To assist the target learning task is to find transferable knowledge across both the networks which are our main focus in the transfer learning scheme. Nodes have the same labels, which have the same structure and either they are connected or tends to be connected closely. Consequently, explore the common structure pattern which can help in author classification in the targeted network ${ }^{36 \mid 38}$. For author classification, our (ACTL) technique is basically comprised of three steps.

Step 1:- Build structure features from both the networks.

Step 2:- Reconstructed the features of the target network from our explored similar signature subgraph by source and target network.

Step 3:- For learning a classifier together in a target network to classifying and reconstruct the feature of author.

A network comprised of a set of nodes and edges, besides each node will describe two types of feature, Content features define the features of the node while the structure feature defines the node with respect to neighborhood structure information $^{39}$. In this work, we defined the structure information of the node based on the author subgraph. First, we explore the major structure patterns of the neighborhood node. Suppose the label of node depend upon its neighborhood structure which is contained by the depth of $s$ for any node $v$ For example, a node $v$ consider as a root node in the network, using a breadth-first search $(B F S)^{47}$ crawl to the neighborhood structure of the node with the depth of $s$. Suppose the $D B L P$ network, we build neighborhood structures for node $A$. We propose a way to represent a uniform level of neighborhood structures based on the similarity of two sub-graphs; furthermore, define by a mapping function $M:(n, s) \longrightarrow n$, describe the structure of node $n$ to make a vector within the depth $s$ of s-neighborhood structures. We able to establish a set of subgraph just in case s-neighborhood structure is organized by means of subgraphs to signify the neighborhood structure. Let we represent the whole space $A=\left(a_{1}, a 2, \ldots, a_{k}\right)$, then based on subgraph we can built a mapping function of $M$. Consequently, The formed values from subgraph-based feature vector $v_{1}, \ldots, v_{k}$ where subgraph based $a_{i}$ will be equivalent feature value of $v_{i}$.

A graph $D=(N, E)$ and the node $(n \in N)$, scan recursively from root node to all neighbor nodes till the depth of node $s$,as well as s-neighborhood structures $d_{n}$ is subtree of node $n$ which comprise of all inventive link and visited nodes. $d=\left(N_{d}, E_{d}\right)$, where, $d$ is subgraph of $D$,

$\forall n \in N_{d}, f(n) \epsilon D$;

$\forall(t, n) \epsilon E_{d},(f(t), f(n)) \epsilon E$.

The graph $D=(N, E)$, subgraph bases $\mathrm{A}=\left\{a_{1}, a_{2}, \ldots, a_{k}\right\}$ and $\left\{v_{1}, \ldots, v_{k}\right\}$ is the group of value where every $V_{i}$ value is relates to $a_{i}$ of subgraph $\mathrm{A}=\left\{a_{1}, a_{2}, \ldots, a_{k}\right\}$; moreover, the graph $D$ as well as author subgraph bases $A$ is exclusively define $D^{\prime}$.

Discover the subgraph bases $A$ and its corresponding values to compute from graph $D$ by means of s- neighborhood structures.

$$
A=\left\{a_{1}, a_{2}, \ldots, a_{k}\right\}=\text { Exclusive }\left\{d_{1}, d_{2}, \ldots, d_{k}\right\}
$$

The association between the author subgraph is establish using s-neighborhood structures $A=a_{1}, a_{2}, \ldots, a_{k}$, in addition to compute the probability value $\operatorname{pr} b\left(d \mid a_{i}\right)$, the probability value $\operatorname{pr} b\left(d \mid a_{i}\right)$ will be large in case the structure of $g$ to be expected the subgraph base $a_{i}$ otherwise, the probability value $\operatorname{pr} b\left(d \mid a_{i}\right)$ will be near to zero.

$$
w=\left\{v_{1}, v_{1}, \ldots, v_{k}\right\}=\operatorname{prb}\left(d \mid a_{1}\right),\left\{\operatorname{prb}\left(d \mid a_{2}\right), \ldots, \operatorname{prb}\left(d \mid a_{k}\right)\right\}
$$

$\operatorname{prb}\left(d \mid a_{i}\right)$ the subgraph base of $a_{i} \epsilon A$ with respect the value of $v_{i}$ to $d$. We describe the mutual subgraph between graph $D_{1}$ and $D_{2}$, besides the graph $D(N, E)$, assume that $|N|$ and $|E|$ represent the sum of nodes and edges. We assume, $|D|$ represent the total sum of nodes and edges of $|D|$ where $D=|N|+|E|$. Compute the probability $\operatorname{prb}\left(d \mid a_{i}\right)$ as follows,

$$
\operatorname{prb}\left(d \mid a_{i}\right)=\frac{\left|\operatorname{Mmutal}\left(d, a_{i}\right)\right|}{M x\left(|d|, \mid a_{i}\right) \mid}
$$

The $\operatorname{prb}\left(d \mid a_{i}\right)$ will be larger if more mutual structures of $d$ with $a_{i}$, while the $\operatorname{prb}\left(d \mid a_{i}\right)$ will be near to 0 in case small mutual structures of $d$ with $a_{i}$. The probability $\operatorname{prb}\left(d \mid a_{i}\right)$ for a known graph $d$ as well as subgraph of $a_{i}$ has 
following features.

$0<\operatorname{prb}\left(d \mid a_{i}\right) \leq 1$

$\operatorname{prb}\left(d \mid a_{i}\right)=1 \longleftrightarrow d$ and $a_{i}$ are Isomorphic, if $d_{1}, d_{2}$ are isomorphic then $\operatorname{prb}\left(d_{1} \mid a_{i}\right)=\operatorname{prb}\left(d_{2} \mid a_{i}\right)$

Extract the useful feature from source to the target network and mine the specifiable patterns from $D_{s}$ to $D_{t}$. Even if the network we have an appropriate domain but it's can be some distinct feature in their node. We have to define an optimal set of mutual subgraph $A_{(k)}^{*}$ to capture mutual pattern between $D_{s}$ and $D_{t}$.

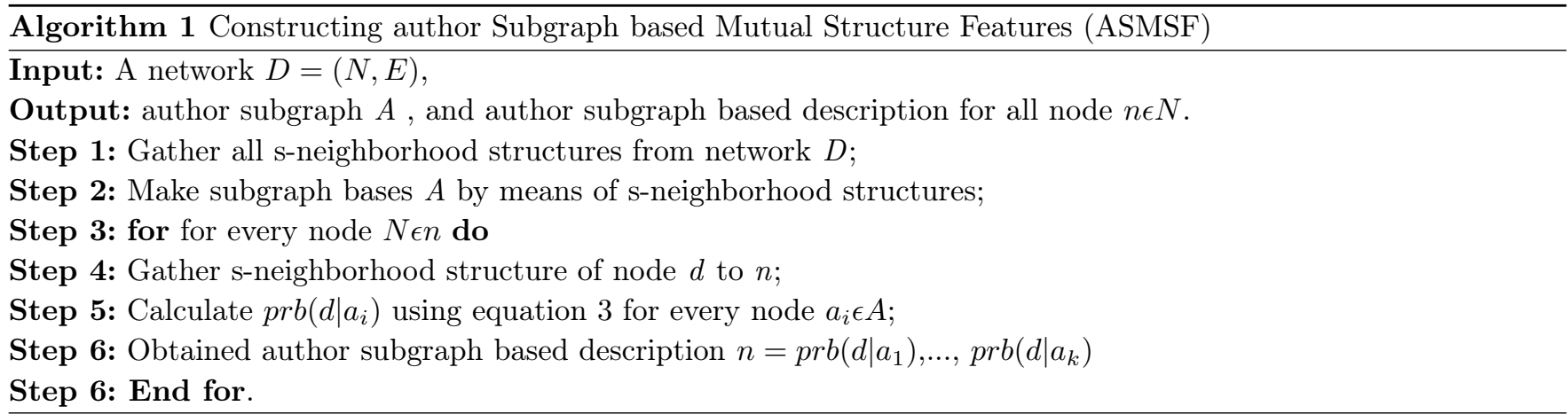

The $A_{s}$ and $A_{t}$ describe as subgraph which based on source network and target network $D_{s}, D_{t}$; consequently, find an optimum level set of mutual subgraph $A_{(k)}^{*}$ which can be select as whole set of $A_{s}$ union $A_{t}$ to signify the source and target networks $\underline{40}$.

$$
\begin{array}{r}
A_{(k)}^{*}=\arg _{A_{(k)}} \max \prod_{n \in D_{t}} \operatorname{prb}\left(d_{n} \mid A_{(k)}\right) \prod_{n \in D_{s}} \operatorname{prb}\left(d_{n} \mid A_{(k)}\right) \\
A_{(k)}^{*}=\arg _{A_{(k)}} \max \\
\prod_{n \in D_{t}} \prod_{a_{i} \in A_{(k)}} \operatorname{prb}\left(d_{n} \mid a_{(i)}\right) \\
\prod_{n \in D_{s}} \prod_{a_{i} \in A_{(k)}} \operatorname{prb}\left(d_{n} \mid a_{(i)}\right)
\end{array}
$$

The $D_{s}$ and $D_{t}$ are source and target network are signified in mutual subgraph to collect unique neighborhood structure ${ }^{4142}$. We describe

$$
F=\prod_{a_{i} \in A_{(k)}}\left\{\prod_{n \in D_{t}} \operatorname{prb}\left(d_{n} \mid a_{(i)}\right) \prod_{n \in D_{s}} \operatorname{prb}\left(d_{n} \mid a_{(i)}\right)\right\}
$$

using in log form,

$$
\log F=\sum_{a_{i} \in A_{(k)}} \log \left\{\prod_{n \in D_{t}} \operatorname{prb}\left(d_{n} \mid a_{(i)}\right) \prod_{n \in D_{s}} \operatorname{prb}\left(d_{n} \mid a_{(i)}\right)\right\}
$$

for optimum description to rewrite the Eq.(5) as

$$
A_{(k)}^{*}=\arg _{A_{(k)}} \max \log F .
$$

Here, to select the optimum set of $a_{i}^{\prime}$ which can be maximize the addition of $\log \left\{\prod_{n \epsilon D_{t}} \operatorname{prb}\left(d_{n} \mid a_{(i)}\right) \prod_{n \epsilon D_{s}} \operatorname{prb}\left(d_{n} \mid a_{(i)}\right)\right\}$.

As $0<\operatorname{prb}\left(d_{n} \mid a_{(i)}\right) \leq 1$

$$
\log \left\{\prod_{n \in D_{t}} \operatorname{prb}\left(d_{n} \mid a_{(i)}\right) \prod_{n \in D_{s}} \operatorname{prb}\left(d_{n} \mid a_{(i)}\right)\right\}<0
$$


for optimum function to rewrite as

$$
\begin{array}{r}
A_{(k)}^{*}=\arg _{A_{k}} \min \sum_{a_{i} \in A_{(k)}}\left\{-\log \left\{\prod_{n \in D_{t}} \operatorname{prb}\left(d_{n} \mid a_{(i)}\right)\right.\right. \\
\left.\left.\prod_{n \in D_{s}} \operatorname{prb}\left(d_{n} \mid a_{(i)}\right)\right\}\right\} .
\end{array}
$$

The source and target $D_{s}$ and $D_{t}$ networks used to calculate $\operatorname{prb}\left(d_{n} \mid a_{(i)}\right)$ for every node; moreover, to select the set of $A_{k}^{*}$ with lowermost value as $-\log \left\{\prod_{n \epsilon D_{t}} \operatorname{prb}\left(d_{n} \mid a_{(i)}\right) \prod_{n \epsilon D_{s}} \operatorname{prb}\left(d_{n} \mid a_{(i)}\right)\right.$.

Here, we will simplify the classification of the target network to construct a precise classifier on the bases of mutual simplifying author subgraph. Every node of the author graph is not independent while also associated with each other and mutually influence to a connected label of adjacency nodes. Mutual influence classification gives an accurate result and expressively improves the performance of classification as compared with traditional classification method that distinctly classifies the node. 25137 .

We consider three form of feature for every node $n$ and $D_{t}$. (1) The attribute value of node $n$ is allied with its modified features of $m_{n}$. (2) Compute the $n^{\prime}$ unique feature representation $x_{n}$ and gather $n^{\prime}$ s-neighborhood structure of $d_{n}$ for specified node $n$ based on mutual subgraph $A_{(k)}^{*}=\left\{a_{1}, a_{2}, \ldots, a_{k}\right\}$

$m_{n}=\left\{v_{1}, v_{2}, \ldots, v_{k}\right\}$,

$=\left\{\operatorname{prb}\left(d_{n} \mid a_{1}\right), \operatorname{prb}\left(d_{n} \mid a_{1}\right), \ldots, \operatorname{prb}\left(d_{n} \mid a_{k}\right)\right\}$

$x_{n}$ is new feature description on node $n$ is used while the target network has unique feature for every node $m_{\text {new }}=(m, x)$.

(3) The aggregation function is used to analyze the relational feature for every node and gather the statistical data of the node labels from its adjacent node of $n$; moreover, any changes on node label will be effected continuously to relational features. The detailed process of author classification using transfer learning describes in algorithm 2 .

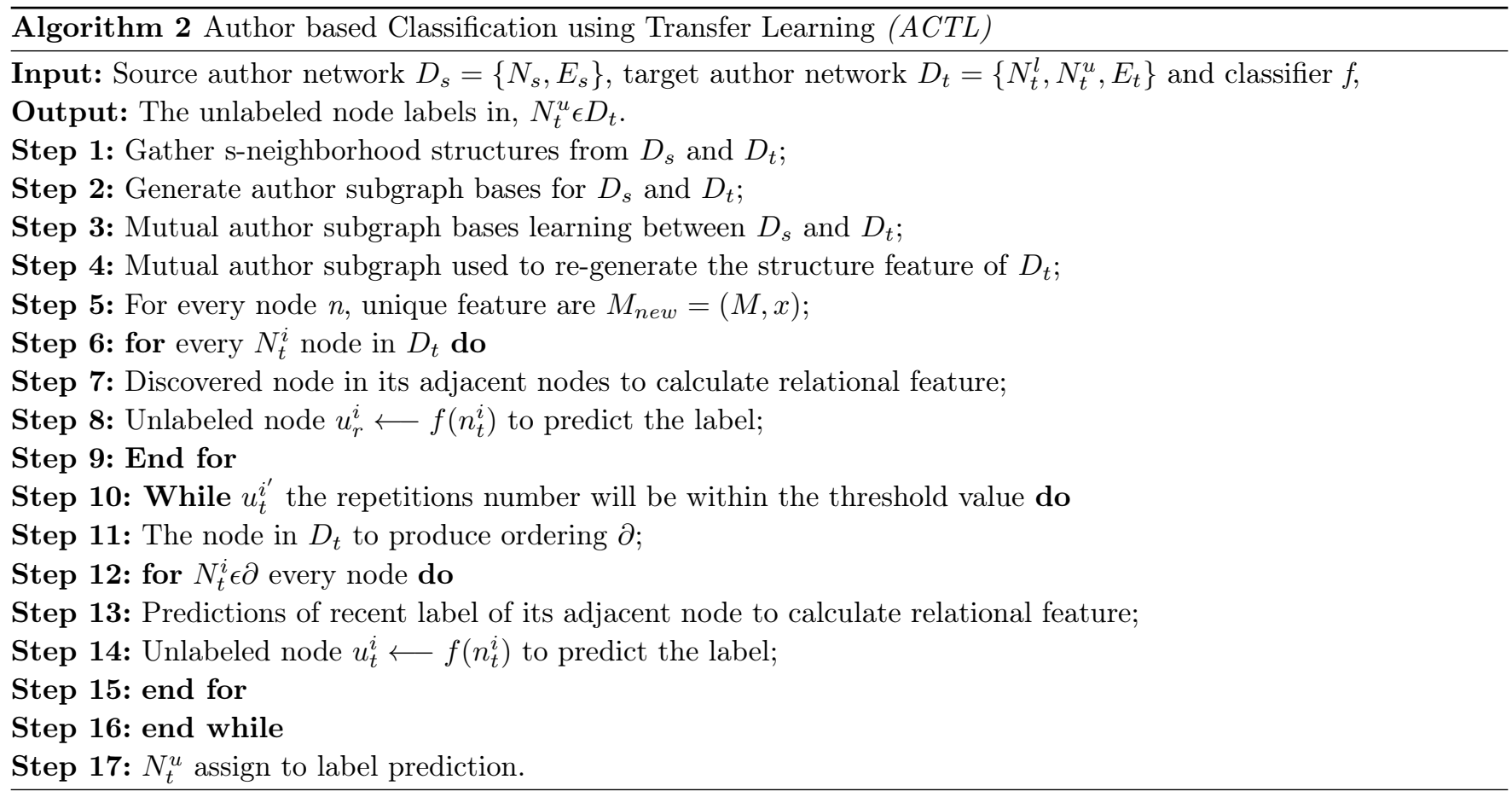

\section{1 | Node-weighting schemes}

Ranking problems in network structure by using the PageRank algorithm to calculate the weighting score of authors. Given a set $\left\{A_{1}, \ldots, A_{n}\right\}$ represents the number of $n$ authors. Calculating the different mutual influence between 
the authors to calculate the ranking score called the rising star score. For mutual influence among the authors, we use $n \times n$ matrix having set $W=\left\{W_{1}, \ldots, W_{n}\right\}$, in set $W$ each value represent the influence between two authors. For instance, $W_{i j}$ represents the mutual influence of author $A_{i}$ over author $A_{j}$. Following is the ranking function to represent the ranking score of $n$ authors.

$$
H\left(A_{i}\right)=\frac{1-d}{n}+d * \sum_{j=1}^{|a|} \frac{\left(A_{i}, A_{j}\right)}{\sum_{k=1}^{|a|}\left(A_{k}, A_{j}\right)} * H\left(A_{j}\right)
$$

$d$ is damping factor, $\left(A_{i}, A_{j}\right)$ is author influence weight to calculate the rank of authors. For finding rising stars score of an author $A_{i}$, we proposed the following main features that are co-authors, corresponding author mutual influence (CAMI), co-author's citations based mutual influence, co-author's venues based mutual influence, co-author's paper-based mutual influence, and co-author's domain and subtopic domain based influence score.

\subsection{1 | Author Influence}

The impact of expert researcher contribution with the new/junior researches is said to be an authors influence. For instance, if a new researcher has started his research with expert author evidently new researcher will get more expertise and more author's influence on those researchers they are presently working with low research profile.

Definition.1 The online social web, the graph $D=(N, E)$, respective user is signified by node $N$ while the mutual influence of the corresponding relationship between two nodes is signified as $E$. To each node $\left(n_{i} \varepsilon N\right)$, where, $i=\{1,2, \ldots, n\}$

$B_{i}$ attributes of user $i$

$B_{i}=\left(b_{i}^{1}, b_{i}^{2}, \ldots, b_{i}^{m}\right)$.

Total number of attribute is $m$ and user $i$, where $j=(1,2, \ldots, m)$

$W_{i j}$ is mutual influence amid two nodes.

\subsection{2 | Corresponding-Author Mutual Influence (CAMI)}

We proposed corresponding authors contribution is based upon mutual influence magnified by co-author contribution and based upon the $s$ index ${ }^{7}$.

If a junior researcher collaborates with senior researchers, then they can gain more score and prominent in future Li et al. 7 .

$$
\text { influnce }\left(C a_{1}, C a_{2}\right)=\frac{\left(C a_{1}, C a_{2}\right)}{P a_{2}}
$$

Total publication of author $C a_{2}$ is $P a_{2}$ where, $c a_{1}$, and $c a_{2}$ are two co-author.

Presume 5 authors are appearing, the first author in name sequence has more contribution comparatively with the last author because the main theme is when a junior author works with experienced researcher has bright chances to become expert in the future on the another hand last researcher weight will be minimum in that paper author list; however, the last author more or equally contributed with the first author if the last author is the corresponding author.

TABLE 1 Corresponding-authors mutual influence

\begin{tabular}{cc}
\hline Authors & Authors order in paper \\
\hline $\mathrm{L}$ & $1(1), 2(1), 3(1), 4(1)$ \\
$\mathrm{M}$ & $1(4), 2(4), 3(4), 4(4)$ \\
$\mathrm{N}$ & $1(2), 2(2), 3(3), 4(3)$ \\
$\mathrm{p}$ & $1(3), 2(3), 3(2), 4(2)$ \\
\hline
\end{tabular}


For example, all four authors are new researcher, author $L$ and $P$ are corresponding-authors and author $L$ and $M$ are co-author in four paper while author $N$ with $P$ and $L$ with $M$ are contributing with each other in two paper shown in Table 1

$$
\begin{gathered}
A S\left(H_{L}, H_{M}\right)=\frac{\left(\sum A S_{L}+\sum A S_{M}\right)}{\sum P A S_{M}} \\
=\frac{(1+1)+(1+0.5)}{1+0.5+0.5+1}=1.05 \\
A S\left(H_{M}, H_{L}\right)=\frac{\left(\sum A S_{M}+\sum A S_{L}\right)}{\sum P A S_{L}}=\frac{(1+0.5)+(1+1)}{1+0.5+1+1}=1 \\
A S\left(H_{N}, H_{P}\right)=\frac{\left(\sum A S_{N}+\sum A S_{P}\right)}{\sum P A S_{P}}=\frac{(0.5+0.5)+(1+1)}{0.5+1+0.33+1}=1.06 \\
A S\left(H_{P}, H_{N}\right)=\frac{\left(\sum A S_{P}+\sum A S_{N}\right)}{\sum P A S_{N}}=\frac{(1+1)+(0.5+0.5)}{0.5+0.5+1+0.2}=1.36
\end{gathered}
$$

$L$ and $N$ are two co-authors in a paper, $A S_{L}$ is author influence score of author $L$; moreover, $P A S_{N}$ is individual weight of author $N$. Author $P$ is the corresponding author with $N$ and cooperate in two papers. Author $N$ naming place is second in both two papers while corresponding author $P$ naming place is third in both two papers but his contribution score is higher than $N$ and he influences more than $N$ even his naming position in that paper is third but always influence weight will be equal with the first author in naming sequence. Earlier schemes, all authors gave same weight or assign weight on the base of naming sequence 1, 2, 3 like first author's contribution is more than the second author and the second author's contribution is more than third author and so on.

\subsection{3 | Venue eminence}

Chronological aspect of an article that is published for a long time ago which get positive chronological association, formulated as ${ }^{10}$ while entropy specify the rank of venues and provide an active score ${ }^{1}$; moreover, imperative venues rank have lower entropy and high entropy scores on an inferior rank of those venues.

$$
\begin{gathered}
\operatorname{TR} W_{\left(H_{i}\right)}=\frac{\sum d\left(H_{i}\right)}{\sum Y_{(d)}} \\
\operatorname{Entropy}(v)=-\sum_{k=1}^{m} W_{k} \log _{2}\left(W_{k}\right) \\
\lambda\left(H_{i}\right)=\frac{1}{|p|} * \sum_{i=1}^{1} \frac{1}{\alpha^{\operatorname{Entropy}(v)}}
\end{gathered}
$$

$T R W\left(H_{i}\right)$ is weight of author $H_{i}$ and $\sum Y_{(d)}$ is age for publication article, $\lambda\left(H_{i}\right)$ is publication score, value of $\alpha$ is $(0<\alpha<1)$

$$
=\frac{1-d}{n}+d * \sum_{j=1}^{|a|} \frac{\operatorname{CAMI}\left(H_{i}\right)}{\sum_{k=1}^{|a|} w\left(H_{k}, H_{j}\right) * \lambda\left(H_{k}\right)}
$$

$w\left(H_{j}, H_{i}\right)$ is influence to other researcher, $n$ is number of all authors, $\lambda\left(H_{i}\right)$ is publication eminence for author $H_{(i)}$.

\subsection{Predicting stars schemes}

\subsection{1 । CAMI based predicting stars finding (CAMIPS)}

PubRank ${ }^{[7}$ proposed from educational social networks to predict rising stars. Here, we have used author influence score as a substitute for static contribution score to identify the future stars.

$$
=\frac{1-d}{n}+d * \sum_{j=1}^{|a|} \frac{w\left(\mu_{H s}^{d}, \mu_{H t}^{d}\right) * \lambda\left(H_{i}\right) * C A M I\left(H_{j}\right)}{\sum_{k=1}^{|a|} w\left(\mu_{H k}^{d}, \mu_{H s}^{d}\right) * \lambda\left(H_{k}\right)}
$$


Algorithm 3 Corresponding-Author Mutual Influence (CAMI)

Input: Input authors data

Output: Authors ranking score

Step 1: Construct $c a_{2}, p a_{2}$ value for each author from author network further using Eq. 12 to reckon $A C M I$ of all author in succession order.

Step 2: Compute entropy (v) by employing Eq. 15

Step 3: Discover the venue importance by using Eq. 16

Step 4: Calculate authors rank using Eq. 4

$n$ is number of whole authors, $d$ is damping factor, value of $d=0.5, \lambda\left(H_{i}\right)$ is publication reputation for author $H t$.

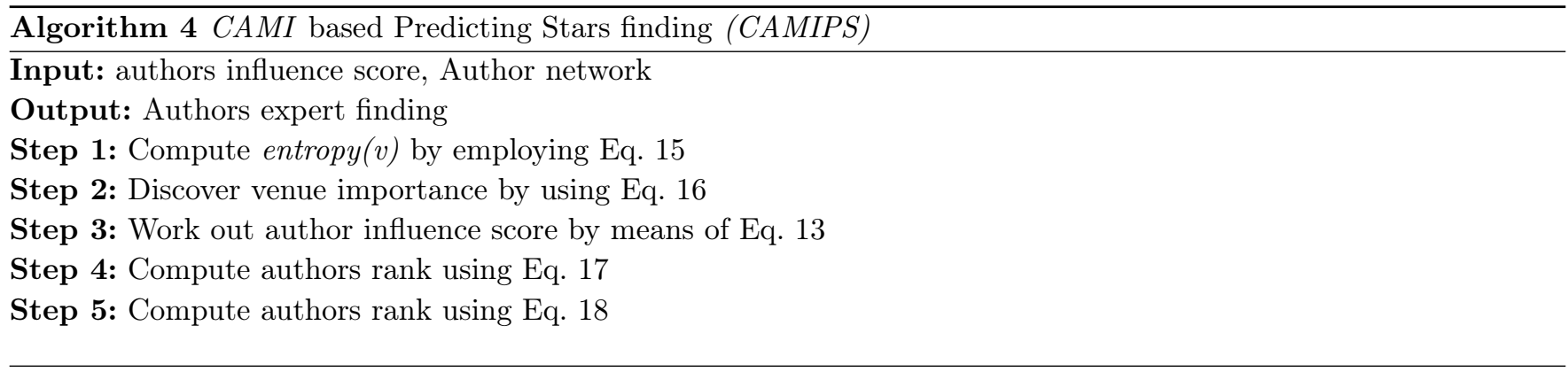

\subsection{2 । Node based Influence score (NIC)}

There are different influence scores on different nodes in vast social networks. Let, node ' $A$ ' has a strong influence on node ' $B$ ' in one case, while in other case nodes ' $B$ ' has a strong influence over node ' $A$ '. The effect of social influence may differ from different angles; for instance, in a research community these types of influences are the collaboration and citations influence of the researchers are being strong or weak on each other's ${ }^{47 / 48}$. For rising stars prediction a small work has been carried out in social networks. Several researchers are collaborating with each other and influenced other researchers in the innumerable cause. The specific topic based social influence identifications using factor graph ${ }^{52 \mid 53}$, the different domains are interrelated on the basis of diverse social influence weight, for example, data mining domain, $A$ has high influence on $B$ but in image processing, $B$ has high influence on $A$, consequently this is very important to know about social influence to segregate with respect to different characteristics. To control the similarity at the topic level for social influence identification is our key attention which is based on the theory of factor graph $\sqrt{52}$, in which the observation data are cohesive on local attributes. The sum-product algorithm is a wait to arrive all the message of the node, so in that case, the algorithm will run in a sequential mode with high complexity. We have adopted an affinity propagation algorithm instead of the factor graph to compute the social influence of the author form two different co-author networks, more detailed presentation is $47 / 48154$.

$$
\begin{gathered}
r_{i j}^{d}=b_{i j}^{d}-\max _{k \in A D(j)}\left\{b_{i k}^{d}+a_{i k}^{d}\right\} \\
a_{j j}^{d}=\max _{k \in A D(j)} \min \left\{r_{k j}^{d}, 0\right\} \\
a_{i j}^{d}=\min \left(\max \left\{r_{j j}^{d}, 0\right\},-\min \left\{r_{j j}^{d}, 0\right\}-\max _{k \in A D(j)}\right. \\
\left.\backslash\{i\} \min \left\{r_{k j}^{d}, 0\right\}\right), i \in A D(j)
\end{gathered}
$$


where $A D(j)$ is adjacent node of $j, d$ is specific domain, $b_{i j}^{d}$ is logarithm normalized function, $r_{i j}^{d}$ refer the influence weight from $i$ to $j$ and $a_{i j}^{d}$ refer the influence weight from $j$ to $i$.

$$
\begin{gathered}
b_{i j}^{d}=\log \frac{\left.g\left(v_{i}, \mathbf{y}_{i}, d\right)\right|_{y_{i}^{d}=j}}{\left.\sum_{k \in A D(i) \cup\{i\}} g\left(v_{i}, \mathbf{y}_{i}, d\right)\right|_{y_{i}^{d}=k}} \\
\mu_{s t}^{d}=\frac{1}{1+e^{-\left(r_{t s}^{d}+a_{t s}^{d}\right)}}
\end{gathered}
$$

$a$ and $r$ are variable, $v_{i}$ and $v_{j}$ are node, while the social influence score based on both variables, $\mu_{s t}^{d}$ is social influence of one node to other on the basis of different domain. The social network is a large network that has millions of users connected with each other. Algorithm. 5 node based influence score (NIC) obtained $\mu_{s t}^{d}$ from large authors network to identify the specific domain based predicting stars.

\footnotetext{
Algorithm 5 Node base Influence score $(N I C)$

Input: Input authors data $G=(V, E)$

Output: Authors domain based influence graph

Step 1: compute feature function $g\left(v_{i}, y_{i}, d\right)$

Step 2: Compute $b_{i j}^{d}$ using Eq. 22

Step 3: Set value $r_{i j}^{d} \longleftarrow 0$

Step 4: Outer loop

Step 5: loop: author topic pair $\left(e_{i j}, d\right)$ do

Step 6: calculate $r_{i j}^{d}$ by using Eq. 19

Step 7: end loop

Step 8: loop: calculate $\left(v_{j}, d\right)$ do

Step 9: update $a_{j j}^{d}$ using to Eq. 20

Step 10: end loop

Step 11: loop $\left(e_{i j}, d\right)$

Step 12: Compute $a_{i j}^{d}$ using Eq. 21

Step 13: end loop

Step 14: outer loop end

Step 15: loop:node $v_{t}$

Step 16: loop: $A D_{s} \in A D(t) \cup t$

Step 17: compute $\mu_{s t}^{d}$ Eq. 23

Step 18: end inner loop

Step 19: end outer loop
}

\subsection{3 । NIC based Predicting Stars finding (NICPS)}

Above algorithm, 3 using PageRank ${ }^{17}$ algorithm to use influence score instead of transition probability to specify the active node from the social network. Here the application on expert identification in social influence graphs, We substitute the PageRank transition probability with the influence score. We consider the influence score as we define instead of traditional PageRank algorithm $\frac{17}{17}$, in which $p\left(v \mid v^{\prime}\right)$ is simply the number of out links of node $p\left(v^{\prime}\right)$.

$p\left(v \mid v^{\prime}\right)$ influence of node

$$
\begin{gathered}
r[v]=\beta \frac{1}{|v|}+(1-\beta) \sum_{v^{\prime}: v^{\prime} \longrightarrow v} r\left[v^{\prime}\right] p\left(v \mid v^{\prime}\right) \\
p\left(v \mid v^{\prime}\right)=\frac{\sum_{d} \mu_{v^{\prime} v}^{d}}{\sum_{v^{\prime}: v^{\prime} \longrightarrow v} \sum_{d} \mu_{v^{\prime} v}^{d}}
\end{gathered}
$$


Algorithm 6 NIC based predicting stars finding (NICPS)

Input: authors influence score, Author data

sqrt Output: Authors stars finding

Step 1: Work out author influence score by means of algorithm 4

Step 2: Stars identify by using Eq. 24

\subsection{4 | Specific Topic Domain based Predicting Stars (STDPS)}

In algorithm 6 defined the co-author relationship between other authors with the same domain to compute the author ranking.

$$
\begin{gathered}
r[v, d]=\beta \frac{1}{|v|} p\left(d_{k} \mid v\right)+(1-\beta) \sum_{v^{\prime}: v^{\prime} \longrightarrow v} r\left[v^{\prime}, d\right] p\left(v \mid v^{\prime}, d\right) \\
p\left(v \mid v^{\prime}, d\right)=\frac{\mu_{v^{\prime} v}^{d}}{\sum_{v_{j^{\prime}}: v^{\prime} \longrightarrow v_{j}} \mu_{v^{\prime} v_{j}}^{d}}
\end{gathered}
$$

$p\left(v \mid v^{\prime}, d\right)$ epitomize the influence of one node $v^{\prime}$ to other node $v$ on specific domain $d ; p(d \mid v)$ is domain $d$ based probability. The each node $v$, a vector of ranking scores $r[v ; d]$, in which each node is specific to topic $d$ while we select the co-author relationship randomly within same topic domain to define the topic based ranking score as: Topic model; $p\left(v \mid v^{\prime}, d\right)$ represents the probability of node $\left(v^{\prime}\right)$ influencing node $v$ on topic $d$ where $p(d \mid v)$ is the probability of topic $d$ generated by node $v$.

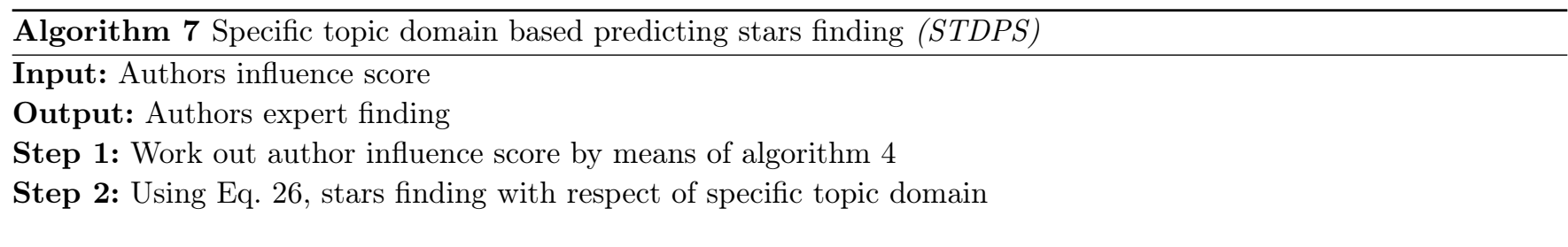

\section{4 | EXPERIMENTS}

To validate our proposed algorithm we extensively described the classification performance and the evaluation of predicting rising stars.

\section{1 | Data sets}

We used three real-world networks in our experiments, signifying Arnetminer, digital bibliography, and library project (DBLP) CORA. Arnetminer contain 38432 publication data, Citeseer contains 3,542, and

emphCora 2,834 contain 5239 publications data 2 156157|58. In our experiment 14 topic domain Machine Learning, Data mining, Databases, Semantic web, Information Retrieval, Human-Computer Interaction, Artificial Intelligence, Web service, Neural Networks, Case-Based, Probabilistic Methods, Rule Learning, Genetic Algorithms, Reinforcement Learning Theory; furthermore, the publications data of journal and conference from 1995 to 2000 are used in our experiment.

We examined that the generated subgraph will be more precise in the target domain on the individual network if the value of $T$ will be large. The total number of node and edges in author subdomain $A$, where $\left|N_{A}\right|+\left|E_{A}\right| \ll N$, time complexity will be less than $O\left|N^{2}\right|$; moreover, the running time mainly affects if the value of $T$ increase in our algorithm besides, it will be more helpful of fair prediction of rising stars. Furthermore, we built one transferring 
knowledge of common author subdomain data-set from two cross networks and chosen authors from each domain that are extremely improved the classification precision in the target domain. In the mutual database, when we used the source domain in the target domain. The similarity is absolutely correlated in the accuracy of classification because, in a scientific publication of both author domain citations, co-authorship, and venue association make highly effective for transfer learning.

\subsection{1 | Impact the Depth of s-Neighborhood Structure}

Classification precision is increased if we select more subgraphs to be transferred thru the target networks $60,59,34,61$. The proposed algorithm outperforms on the distinct number of $k$ subgraphs, as well as the highest value of $k$ subgraph found very complex in source network. We analyze the influence of depth s-neighborhood structure and classification accuracy for nodes. If the value of $s$ is one then we only take the immediate neighbor node. The number of nodes can rapidly increase of s-neighborhood structures if we recursively move the adjacent of a nodeąís adjacent. We change the depth of $s$ values from 1 to 10,1 to 20 to make s-neighborhood structures for distinct network and different subgraphs to find the mutual subgraph between source and target networks.

\section{2 $\mid$ Performance Evaluation of Predicted Rising Stars}

In this area there is a small amount of work is done to predict the rising stars. Its important challenge is to mine the novel scholars who will be a star in forthcoming. The first time we are using transfer learning to predict the target domain classification to predict and differentiate the difference between the rising stars, well established, stable and declining authors that revealed in Figure. 12 .

\subsection{1 | Rising Stars}

Rising stars are said to be those persons/researchers who have currently low profile or who may not be in the top in their respective areas but they can be a star in the future. It is a kind of prediction in which a new person/research who is new to their respective field and can be a star in the future upon his features. Features are basically the impact of different factors on the new author which makes him/her a rising star e.g. if an author is working under the supervision of already experienced and have high profile authors have a high impact on new authors to become a predicting star. Predicting rising stars is one of the important and useful in different fields as if we appoint a young faculty member. This technique plays a vital role to appoint a rising star which will be definitely beneficial for the department or selecting reviewers for the journal, shown in Figure. 3 .

\subsection{2 | Author identifications analysis}

In this section, we identified and differentiated the difference between the rising stars, well established, stable and declining stars. We have calculated authors score and authors influenced score that highly influenced the rank of final author scores.

We recognized those authors they were not predicting rising stars 1178 . According to the above definition in Fig. 3 . they are already well established, stable and declining authors whom we will not consider in predicting rising stars, as well as they; declared predicting stars in the previous methods 11178 . Furthermore, consider only those authors who will be in the span of 1995 to 2000.

\subsection{3 | Baseline Method evolution}

The PubRank ${ }^{17}$ predicted a 10 rising stars from database domain, StarRank ${ }^{1}$ predicted 10 rising stars, and author ${ }^{8}$ predicted 30 predicting rising stars shown in Table.2[3|4. We have individually analyzed each author score sequentially with specific domain based on influence score, and mine 30 authors. Proposed algorithm give fair authors rank to respective authors and identified the rising stars, well established, stable, and declining authors which is ascertained of our algorithm, shown in Table. 2|3|4, despite the fact that more detail in Example. 1.

Mahmut T. Kandemir is declining author, he published 2004 (1, 53), 2005(1, 10), 2006(2, 62) čň2014 (10, 65), $2015(11,55)$ čň2016 $(14,45)$ research article. The author has 1 publication in 2004 and got 53 citation, in 2005 have 
TABLE 2 Performance comparison of Predicting rising stars using 1st data-set

\begin{tabular}{|c|c|c|c|c|c|c|}
\hline & Author Name & PubRank ${ }^{17]}$ & StarRank $^{1}$ & 8 & Proposed & $\mathrm{AS}$ \\
\hline 1 & Moses Charikar & NA & NA & Rising Star & Rising Star & 1.59 \\
\hline 2 & Ravi Kumar & NA & NA & Rising Star & Rising Star & 1.81 \\
\hline 3 & Rajeev Alur & NA & NA & Rising Star & Well Established & 2.21 \\
\hline 4 & B.F. F.Ouellette & NA & NA & Rising Star & Well Established & 1.05 \\
\hline 5 & Amit Sahai & $\mathrm{NA}$ & NA & Rising Star & Rising Star & 1.54 \\
\hline 6 & Ee-Peng Lim & NA & NA & Rising Star & Well Established & 1.12 \\
\hline 7 & S. Rajagopalan & $\mathrm{NA}$ & $\mathrm{NA}$ & Rising Star & Well Established & 1.22 \\
\hline 8 & Hari Balakrishnan & NA & NA & Rising Star & Well Established & 2.64 \\
\hline 9 & Sudipto Guha & NA & NA & Rising Star & Well Established & 1.32 \\
\hline 10 & Sonia Fahmy & NA & NA & Rising Star & Well Established & 0.48 \\
\hline 11 & Mahmut T. Kandemir & NA & NA & Rising Star & Declining Author & 0.08 \\
\hline 12 & Barbara A. Rapp & NA & NA & Rising Star & Well Established & 0.73 \\
\hline 13 & Byron Dom & NA & NA & Rising Star & Well Established & 1.09 \\
\hline 14 & V. Guruswami & NA & NA & Rising Star & Rising Star & 1.08 \\
\hline 15 & S. Chakrabarti & NA & NA & Rising Star & Well Established & 1.28 \\
\hline 16 & Chandra Chekuri & NA & NA & Rising Star & Rising Star & 1.13 \\
\hline 17 & David L. Wheeler & NA & NA & Rising Star & Well Established & 2.44 \\
\hline 18 & S. Kalyanaraman & NA & NA & Rising Star & Well Established & 0.92 \\
\hline 19 & Ian Horrocks & NA & NA & Rising Star & Rising Star & 1.88 \\
\hline 20 & Rajeev Rastogi & Rising Star & NA & Rising Star & Well Established & 1.47 \\
\hline 21 & Thad Starner & NA & NA & Rising Star & Well Established & 1.29 \\
\hline 22 & Wee Keong Ng & NA & NA & Rising Star & Stable & 0.19 \\
\hline 23 & David J. Lipman & NA & NA & Rising Star & Well Established & 2.58 \\
\hline 24 & Michael A. Bender & NA & NA & Rising Star & Well Established & 0.35 \\
\hline 25 & Srinivasan Seshan & NA & NA & Rising Star & Well Established & 1.30 \\
\hline 26 & Jeen Broekstra & NA & NA & Rising Star & Declining Author & 0.41 \\
\hline 27 & George Karypis & NA & NA & Rising Star & Well Established & 0.52 \\
\hline 28 & Gonzalo Navarro & NA & NA & Rising Star & Rising Star & 1.06 \\
\hline 29 & Steven D. Gribble & $\mathrm{NA}$ & NA & Rising Star & Well Established & 1.24 \\
\hline 30 & Erik D. Demaine & $\mathrm{NA}$ & Rising Star & Rising Star & Rising Star & 1.13 \\
\hline 31 & Wei Ying Ma & NA & Rising Star & NA & Rising Star & 1.69 \\
\hline 32 & Philip S. yu & Rising Star & Rising Star & $\mathrm{NA}$ & Well Established & 2.44 \\
\hline 33 & Jiawei Han & Rising Star & Rising Star & NA & Well Established & 2.60 \\
\hline 34 & Zheng Chen & NA & Rising Star & NA & Well Established & 0.85 \\
\hline 35 & Divesh Srivastava & NA & Rising Star & NA & Well Established & 1.19 \\
\hline 36 & Wei Wang & NA & Rising Star & $\mathrm{NA}$ & Well Established & 1.20 \\
\hline 37 & Hsinchun Chen & NA & Rising Star & NA & Well Established & 1.27 \\
\hline 38 & Bertram Ludscher & NA & Rising Star & $\mathrm{NA}$ & Well Established & 0.39 \\
\hline 39 & Lee Tan & NA & Rising Star & NA & Declining Author & 0.01 \\
\hline 40 & B. K. Bhargava & Rising Star & $\mathrm{NA}$ & NA & Stable & 0.12 \\
\hline 41 & H. V. Jagadish & Rising Star & NA & NA & Well Established & 1.18 \\
\hline 42 & Hamid Pirahesh & Rising Star & NA & $\mathrm{NA}$ & Well Established & 1.03 \\
\hline 43 & Ming-Syan Chen & Rising Star & NA & NA & Well Established & 1.24 \\
\hline 44 & Rakesh Agrawal & Rising Star & NA & $\mathrm{NA}$ & Well Established & 1.01 \\
\hline 45 & Richard R. Muntz & Rising Star & NA & NA & Well Established & 1.09 \\
\hline 46 & Shi-Kuo Chang & Rising Star & NA & NA & Well Established & 10.35 \\
\hline
\end{tabular}



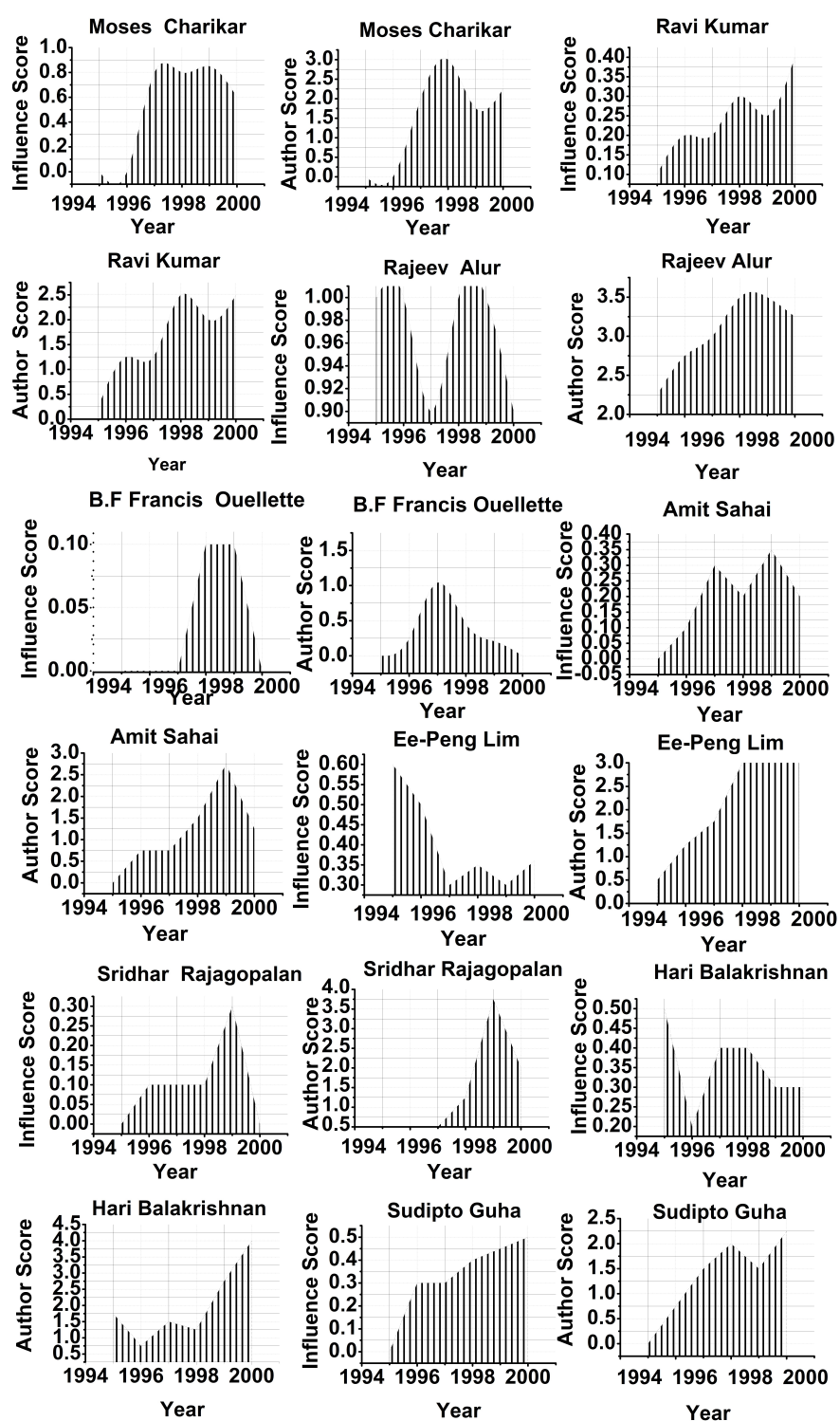

FIGURE 1 Performance evaluation of rising stars, well established, stable and declining authors(First Data set)

1 publication and got 10 citations, in 2006 have 2 publications and got 62 citations even though have no publication between 1995 to 2000, he had declared predicting star in the previous method. The author Rakesh Agrawal is a well-established author. He published a research article in 1965(1,0), 1970(3.0), 1979(2,3), 1980(8,0), 1981(3,0), 1982(1,0)čň1983(6,97), 1984(4,1), 1985(12,739), 1986(4,0), 1987(12,964), 1988(7,450), 1989(14,1010), 1990(11,329), 1991(22,574), 1992(10,352), 1993(28,22327), 1994(14, 22853). He has 1 publication and 0 citations in 1965, 3 research publications and 0 citations in 1970, and so on. Jiawei Han is a well-established author. He published in 1985(2, 14), 1986(1, 41), 1987(1, 37), 1988(6,107), 1989(4, 63), 1990(4, 96), 19991(9,368), 1992(9,709), 1993(14,302), 1994(18, $5640)$ research article. He has 2 publications and 14 citations in 1985, in 1978 has 1 publication and 37 citations, and so on.

Philip S. yu is a well-established author. He published in 1977(3,28), 1978(1, 0)čň1981(1, 10), 1983(2, 7), 1985(3, 120), 1986(7, 120), 1987(7, 665), 1988(7, 72), 1989(14, 1010),1990(13, 626), 1991(14, 985), 1992(24, 999), 1993(18, 1209), 1994(22, 770). He has 3 publications in 1977 and got 28 citations, 1 research article and got 0 citations in 1978, and so on. Jianbo Shi is a well-established author. He has published in 1993 (2, 8273), 1994(1, 0). He has 2 publication in 1993 and got 8273 citations and so on. David J. Lipman is a well-established author. He has published 

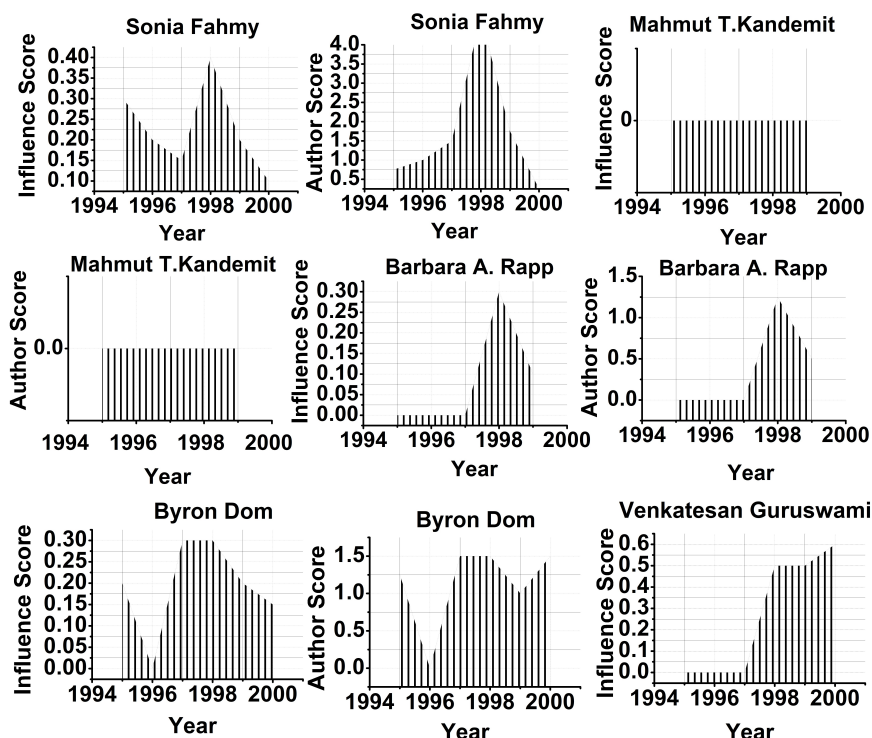

Venkatesan Guruswami

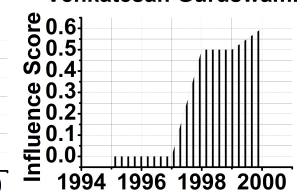

Year

Year

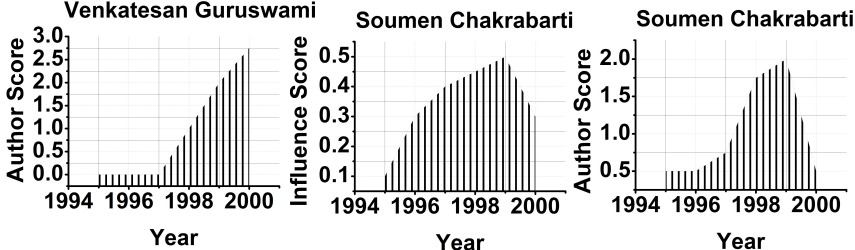

Year
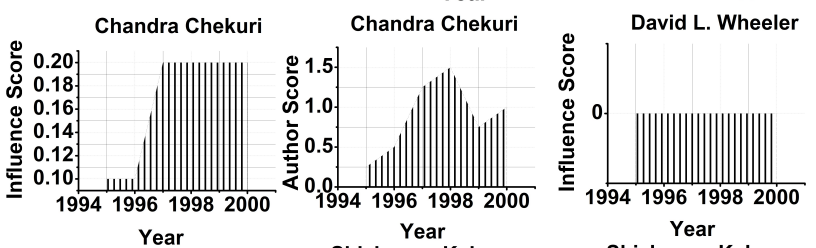

d L. Wheeler Shivkumar Kalyanaraman Shivkumar Kalyanaraman
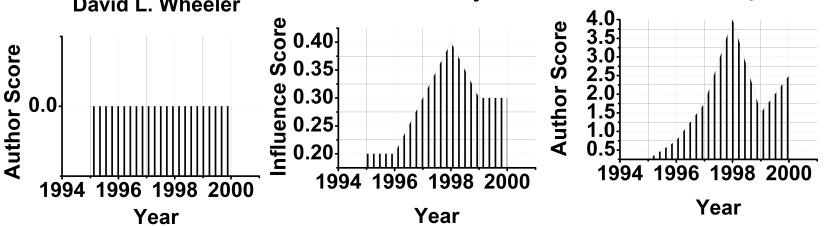

FIGURE 2 Performance evaluation of rising stars, well established, stable and declining authors(Second Data set)

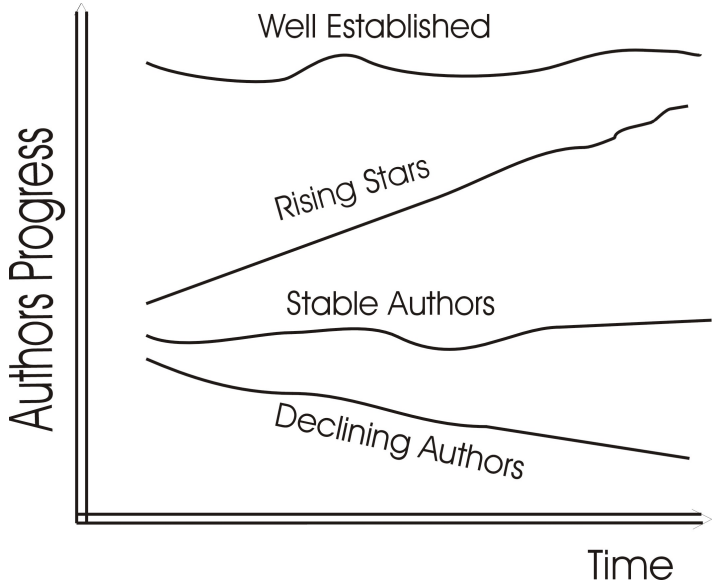

FIGURE 3 Predicting rising star definition, author evolution Tsatsaronis et al ${ }^{62}$ 

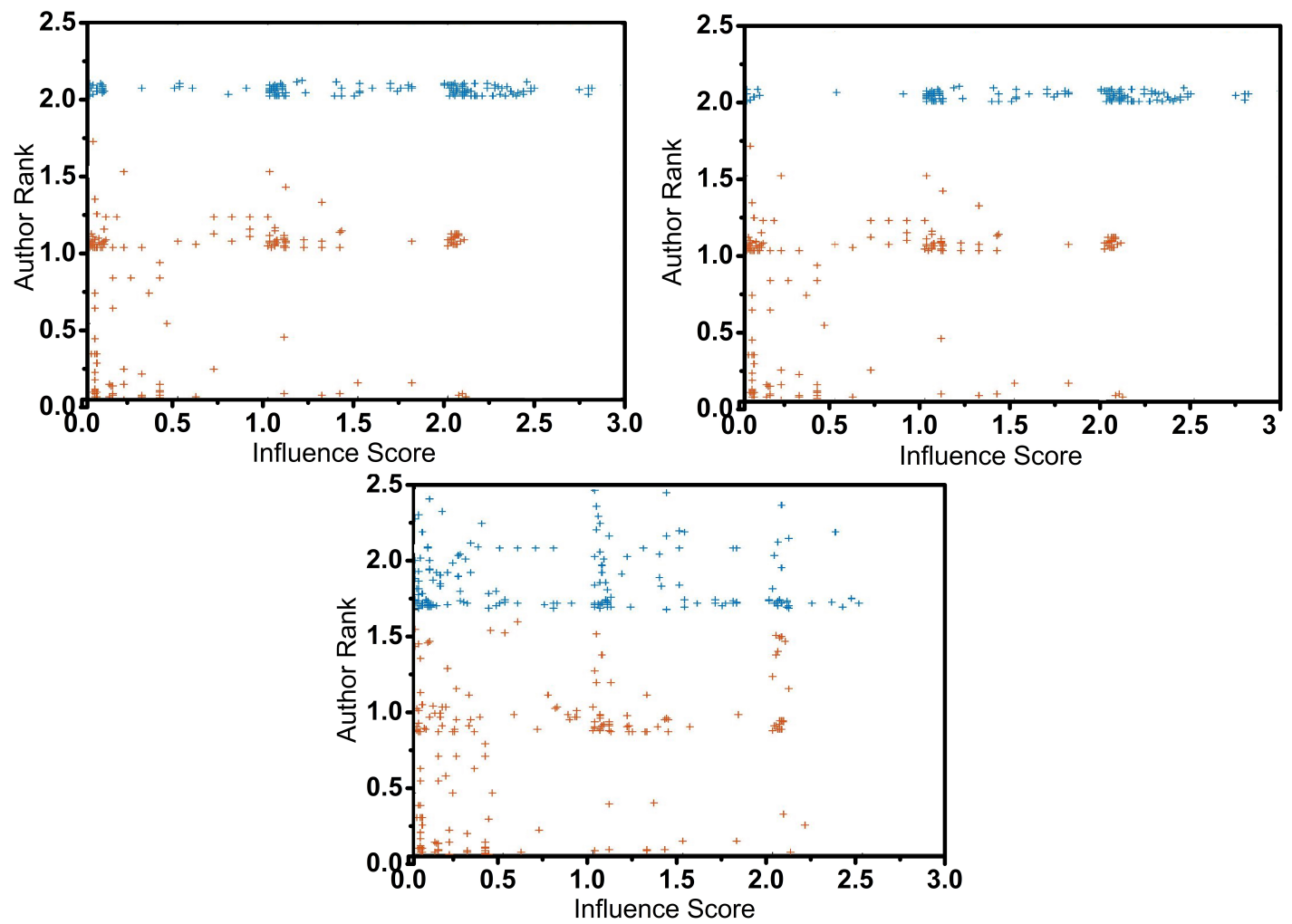

FIGURE 4 Predicting upcoming rising stars

in 1979(1, 0), 1982(2, 6), 1983(1, 0)čň1984(2, 116), 1985(1, 12), 1987(1, 12), 1988(1, 12743), 1989(2, 428), 1990 (5, 69600) 1991(2, 1130), 1993(2, 4428). He has 1 publication in 1983 and got 0 citations, in 1984 he published 2 research articles and got 116 citations, and so on. Michel C. A. Klein is declining author, he published $2000(3,135)$ research article. The author has 3 publication in 2004 and got 135 citations and have no any more research article in the span of 1995 to 2000. Evgeni M. Zdobnov is declining author, he published 2000 (3, 579) research article. The author has 3 publication in 2004 and got 579 citations and have no any more research article in the span of 1995 to 2000.

\section{$4.3 \quad$ Result and discussion}

We revealed the efficiency of proposed techniques CAMIPS, STDPS, NICPS and nodes characteristics for identification on co-authors and citations data set. The previous method selected the representative nodes because of high citation numbers. Proposed algorithms identify the influence between papers; consequently, it can differentiate the citations of academic background of a paper and odd citations. The influenced representative researchers, as well as some influence representative papers, are shown in Table. 566. Preceding methods used to estimate the similar influence score according to similarity-based cosine metric to analyze the social influence of online communities $\frac{54}{54}$ Our method has many different advantages over the similarity-based method. Previous methods can only calculate the similarity between the nodes but cannot provide any information or data regarding the influence of node on each other.

Using the list of top-30 authors are presented in descending order while all authors have an excellent google scholar profile having a high citation of each paper, besides impressive publication groups like ACM, IEEE, AAAS, and NSERCC SIAM. Furthermore, they also have achieved in different top-level forums like IBM Outstanding Technical Achievement Awards, IBM Canada Research Impact of the Year Award, IBM Outstanding Innovation Award, and IBM Outstanding Innovation Award. They are experts and predicted rising stars during the period of $1995-2000$. If some of them have not become an expert from our top-30 list but still they are rising stars by our method for the reason that there may be several different causes to not continue his research like starting university career, work 
TABLE 3 Performance comparison of Predicting rising stars using second data-set

\begin{tabular}{|c|c|c|c|c|c|c|}
\hline & Author Name & PubRank ${ }^{17}$ & StarRank $^{1}$ & 8 & Proposed & AS \\
\hline 1 & Vahid Tarokh & $\mathrm{NA}$ & NA & Rising Star & Well Established & 0.31 \\
\hline 2 & Ewan Birney & NA & NA & Rising Star & Well Established & 0.26 \\
\hline 3 & B.A. R.Neto & $\mathrm{NA}$ & $\mathrm{NA}$ & Rising Star & Rising Star & 0.25 \\
\hline 4 & Thorsten Joachims & NA & NA & Rising Star & Well Established & 0.34 \\
\hline 5 & Jianbo Shi & $\mathrm{NA}$ & $\mathrm{NA}$ & Rising Star & Well Established & 0.27 \\
\hline 6 & Hamid Jafarkhani & NA & NA & Rising Star & Well Established & 0.21 \\
\hline 7 & Hari Balakrishnan & NA & NA & Rising Star & Well Established & 0.41 \\
\hline 8 & E.L.L.Sonnhammer & NA & NA & Rising Star & Well Established & 0.31 \\
\hline 9 & B. F. F. Ouellette & NA & NA & Rising Star & Well Established & 0.15 \\
\hline 10 & Ian Horrocks & $\mathrm{NA}$ & NA & Rising Star & Rising Star & 0.30 \\
\hline 11 & Dieter Fox & $\mathrm{NA}$ & NA & Rising Star & Well Established & 0.29 \\
\hline 12 & Nello Cristianini & NA & NA & Rising Star & Well Established & 0.27 \\
\hline 13 & S.Rajagopalan & $\mathrm{NA}$ & $\mathrm{NA}$ & Rising Star & Well Established & 0.18 \\
\hline 14 & Steve Lawrence & NA & NA & Rising Star & Well Established & 0.19 \\
\hline 15 & Chris Stauffer & $\mathrm{NA}$ & NA & Rising Star & Rising Star & 0.16 \\
\hline 16 & Mark Handley & NA & NA & Rising Star & Well Established & 0.26 \\
\hline 17 & Keith A. Crandall & NA & NA & Rising Star & Well Established & 0.17 \\
\hline 18 & Ravi Kumar & $\mathrm{NA}$ & $\mathrm{NA}$ & Rising Star & Rising Star & 0.22 \\
\hline 19 & Robert Cooley & NA & NA & Rising Star & Rising Star & 0.13 \\
\hline 20 & Eckart Zitzler & NA & NA & Rising Star & Rising Star & 0.18 \\
\hline 21 & Rajeev Rastogi & Rising Star & NA & Rising Star & Well Established & 0.21 \\
\hline 22 & David J. Lipman & NA & NA & Rising Star & Well Established & 0.32 \\
\hline 23 & P. J.Phillips & NA & NA & Rising Star & Well Established & 0.20 \\
\hline 24 & George Karypis & NA & NA & Rising Star & Well Established & 0.21 \\
\hline 25 & E.M.B-Royer & NA & NA & Rising Star & Declining Author & 0.22 \\
\hline 26 & Iftach Nachman & $\mathrm{NA}$ & NA & Rising Star & Rising Star & 0.14 \\
\hline 27 & Byron Dom & NA & NA & Rising Star & Well Established & 0.17 \\
\hline 28 & Patrick J. Rauss & NA & NA & Rising Star & Rising Star & 0.13 \\
\hline 29 & Hendrik Blockeel & NA & NA & Rising Star & Rising Star & 0.15 \\
\hline 30 & Sudipto Guha & $\mathrm{NA}$ & $\mathrm{NA}$ & Rising Star & Well Established & 0.26 \\
\hline 31 & Wei Ying Ma & NA & Rising Star & NA & Rising Star & 0.34 \\
\hline 32 & Philip S. yu & Rising Star & Rising Star & NA & Well Established & 0.61 \\
\hline 33 & Jiawei Han & Rising Star & Rising Star & NA & Well Established & 0.75 \\
\hline 34 & Zheng Chen & NA & Rising Star & NA & Well Established & 0.14 \\
\hline 35 & Divesh Srivastava & NA & Rising Star & NA & Well Established & 0.27 \\
\hline 36 & Wei Wang & NA & Rising Star & NA & Well Established & 0.26 \\
\hline 37 & Hsinchun Chen & NA & Rising Star & NA & Well Established & 0.32 \\
\hline 38 & B.ludaumlscher & NA & Rising Star & NA & Well Established & 0.17 \\
\hline 39 & Lee Tan & NA & Rising Star & NA & Declining Author & 0.02 \\
\hline 40 & B.K.Bhargava & Rising Star & NA & NA & Stable & 0.04 \\
\hline 41 & H. V. Jagadish & Rising Star & NA & NA & Well Established & 0.33 \\
\hline 42 & Hamid Pirahesh & Rising Star & NA & NA & Well Established & 0.18 \\
\hline 43 & Ming-Syan Chen & Rising Star & NA & NA & Well Established & 0.24 \\
\hline 44 & Rakesh Agrawal & Rising Star & NA & NA & Well Established & 0.20 \\
\hline 45 & Richard R. Muntz & Rising Star & NA & NA & Well Established & 0.22 \\
\hline 46 & Shi-Kuo Chang & Rising Star & NA & NA & Well Established & 0.05 \\
\hline 47 & Erik D. Demaine & NA & Rising Star & NA & Rising Star & 0.20 \\
\hline
\end{tabular}


TABLE 4 Performance comparison of Predicting rising stars using Database Domain

\begin{tabular}{|c|c|c|c|c|c|c|}
\hline & Author Name & PubRank ${ }^{17}$ & StarRank ${ }^{1}$ & 8 & Proposed & AS \\
\hline 1 & Ravi Kumar & $\mathrm{NA}$ & $\mathrm{NA}$ & Rising Star & Rising Star & 1.89 \\
\hline 2 & Moses Charikar & $\mathrm{NA}$ & $\mathrm{NA}$ & Rising Star & Rising Star & 1.79 \\
\hline 3 & B.A. Rapp & $\mathrm{NA}$ & $\mathrm{NA}$ & Rising Star & Well Established & 0.85 \\
\hline 4 & D.L. Wheeler & $\mathrm{NA}$ & $\mathrm{NA}$ & Rising Star & Well Established & 2.49 \\
\hline 5 & B.F.F.Ouellette & NA & NA & Rising Star & Well Established & 0.80 \\
\hline 6 & Rohit Goyal & NA & NA & Rising Star & Stable & 0.10 \\
\hline 7 & Sonia Fahmy & $\mathrm{NA}$ & $\mathrm{NA}$ & Rising Star & Well Established & 0.51 \\
\hline 8 & Amit Sahai & $\mathrm{NA}$ & $\mathrm{NA}$ & Rising Star & Rising Star & 1.61 \\
\hline 9 & V.Guruswami & $\mathrm{NA}$ & $\mathrm{NA}$ & Rising Star & Rising Star & 1.12 \\
\hline 10 & Rajeev Alur & NA & NA & Rising Star & Well Established & 2.28 \\
\hline 11 & S.Rajagopalan & $\mathrm{NA}$ & $\mathrm{NA}$ & Rising Star & Well Established & 1.23 \\
\hline 12 & E. M. Zdobnov & NA & $\mathrm{NA}$ & Rising Star & Declining Author & 0.13 \\
\hline 13 & S.Kalyanaraman & $\mathrm{NA}$ & $\mathrm{NA}$ & Rising Star & Well Established & 0.99 \\
\hline 14 & Ian Horrocks & $\mathrm{NA}$ & $\mathrm{NA}$ & Rising Star & Rising Star & 1.93 \\
\hline 15 & E.D. Demaine & $\mathrm{NA}$ & $\mathrm{NA}$ & Rising Star & Rising Star & 1.20 \\
\hline 16 & M.T. Kandemir & $\mathrm{NA}$ & $\mathrm{NA}$ & Rising Star & Declining Author & 0.09 \\
\hline 17 & Jeen Broekstra & NA & NA & Rising Star & Declining Author & 0.50 \\
\hline 18 & Michael A. Bender & NA & $\mathrm{NA}$ & Rising Star & Well Established & 0.41 \\
\hline 19 & S.Chakrabarti & $\mathrm{NA}$ & $\mathrm{NA}$ & Rising Star & Well Established & 1.33 \\
\hline 20 & Srinivasan Seshan & $\mathrm{NA}$ & $\mathrm{NA}$ & Rising Star & Well Established & 1.38 \\
\hline 21 & Chandra Chekuri & NA & NA & Rising Star & Rising Star & 1.15 \\
\hline 22 & Byron Dom & $\mathrm{NA}$ & $\mathrm{NA}$ & Rising Star & Well Established & 0.22 \\
\hline 23 & M.C. A. Klein & $\mathrm{NA}$ & $\mathrm{NA}$ & Rising Star & Declining Author & 0.05 \\
\hline 24 & Ayman F. Naguib & NA & NA & Rising Star & Declining Author & 0.03 \\
\hline 25 & Wee Keong Ng & $\mathrm{NA}$ & $\mathrm{NA}$ & Rising Star & Stable & 0.24 \\
\hline 26 & David J. Lipman & $\mathrm{NA}$ & $\mathrm{NA}$ & Rising Star & Well Established & 2.61 \\
\hline 27 & Bettina Kemme & NA & NA & Rising Star & Declining Author & 0.53 \\
\hline 28 & Fulvio Corno & $\mathrm{NA}$ & $\mathrm{NA}$ & Rising Star & Stable & 0.34 \\
\hline 29 & Stephan Tobies & NA & NA & Rising Star & Stable & 0.45 \\
\hline 30 & Hari Balakrishnan & $\mathrm{NA}$ & $\mathrm{NA}$ & Rising Star & Well Established & 2.57 \\
\hline 31 & Wei Ying Ma & NA & Rising Star & NA & Rising Star & 1.73 \\
\hline 32 & Philip S. yu & Rising Star & Rising Star & $\mathrm{NA}$ & Well Established & 2.39 \\
\hline 33 & Jiawei Han & Rising Star & Rising Star & NA & Well Established & 2.65 \\
\hline 34 & Zheng Chen & NA & Rising Star & NA & Well Established & 0.94 \\
\hline 35 & Divesh Srivastava & NA & Rising Star & $\mathrm{NA}$ & Well Established & 1.25 \\
\hline 36 & Wei Wang & $\mathrm{NA}$ & Rising Star & $\mathrm{NA}$ & Well Established & 1.24 \\
\hline 37 & Hsinchun Chen & $\mathrm{NA}$ & Rising Star & $\mathrm{NA}$ & Well Established & 1.31 \\
\hline 38 & B.ludaumlscher & $\mathrm{NA}$ & Rising Star & $\mathrm{NA}$ & Well Established & 0.42 \\
\hline 39 & Lee Tan & NA & Rising Star & NA & Declining Author & 0.01 \\
\hline 40 & B.K.Bhargava & Rising Star & NA & NA & Stable & 0.13 \\
\hline 41 & H. V. Jagadish & Rising Star & NA & NA & Well Established & 1.19 \\
\hline 42 & Hamid Pirahesh & Rising Star & $\mathrm{NA}$ & $\mathrm{NA}$ & Well Established & 1.09 \\
\hline 43 & Ming-Syan Chen & Rising Star & $\mathrm{NA}$ & $\mathrm{NA}$ & Well Established & 1.27 \\
\hline 44 & Rakesh Agrawal & Rising Star & NA & NA & Well Established & 1.08 \\
\hline 45 & Richard R. Muntz & Rising Star & $\mathrm{NA}$ & $\mathrm{NA}$ & Well Established & 1.12 \\
\hline 46 & Shi-Kuo Chang & Rising Star & NA & NA & Well Established & 0.39 \\
\hline 47 & Rajeev Rastogi & Rising Star & $\mathrm{NA}$ & $\mathrm{NA}$ & Well Established & 1.49 \\
\hline 48 & Erik D. Demaine & $\mathrm{NA}$ & Rising Star & $\mathrm{NA}$ & Rising Star & 1.16 \\
\hline
\end{tabular}


TABLE 5 Nodes discovering

\begin{tabular}{|c|c|c|}
\hline Dataset & Topics & Nodes \\
\hline Authors & Machine Learning & $\begin{array}{c}\text { Vasant Honavar, Thomas G. Dietterich, } \\
\text { Tom M. Mitchell, Pat Langley, Luc De Raedt, } \\
\text { Zhihua Zhou, Raymond J. Mooney, Ryszard S. Michalski }\end{array}$ \\
\hline- & Data mining & $\begin{array}{c}\text { jiawei Han, Philip s.yu, Rakesh Agrawal, } \\
\text { John C. Shafer, Qiang Yang, Bing Liu, Christos } \\
\text { Faloutsos, Jian Pei, Charu C. Aggarwal, } \\
\text { Vipin Kumar, Xindong Wu, Wei Wang }\end{array}$ \\
\hline- & Database System & $\begin{array}{c}\text { David J. DeWitt, Michael J. Carey, } \\
\text { jiawei Han, Shamkant B. Navathe, Michael Stonebraker, } \\
\text { Philip s.yu, Jennifer Widom, Jeffrey D. Ullman, }\end{array}$ \\
\hline- & Semantic Web & $\begin{array}{c}\text { Steffen Staab, James Hendler, Amit P. Sheth, } \\
\text { Tim Finin, Dieter Fensel, Frank Van Harmelen } \\
\text { Deborah L. Mcguinness, Rudi Studer, } \\
\text { Andrew Tomkins, Tim Berners-Lee }\end{array}$ \\
\hline - & Information Retrieval & $\begin{array}{c}\text { W. Bruce Croft, Gerard Salton, } \\
\text { Susan T. Dumais, Justin Zobel, James Allan, Nicholas J. Belkin } \\
\text { Alan F. Smeaton, James P. Callan, Maarten De Rijke }\end{array}$ \\
\hline- & Web Service & $\begin{array}{c}\text { Boualem Benatallah, Sheila Mcilraith, Fabio Casati, Carole Goble } \\
\text { Geoffrey C. Fox, Schahram Dustdar }\end{array}$ \\
\hline Citation & Machine Learning & $\begin{array}{c}\text { Ensemble methods in machine learning } \\
\text { Combining labeled and unlabeled data with co-training }\end{array}$ \\
\hline- & Data mining & $\begin{array}{l}\text { Fast algorithms for mining association rules } \\
\text { Mining association rules between sets of items in large databases }\end{array}$ \\
\hline- & Database System & $\begin{array}{c}\text { The Object-Oriented Database System Manifesto } \\
\text { Data Mining: An Overview from a Database Perspective }\end{array}$ \\
\hline- & Semantic Web & $\begin{array}{l}\text { The Semantic Web, OWL Web Ontology Language Overview } \\
\text { Knowledge engineering: principles and methods }\end{array}$ \\
\hline- & Information Retrieval & $\begin{array}{c}\text { Introduction to Modern Information Retrieval,Information retrieval } \\
\text { Indexing by latent semantics analysis }\end{array}$ \\
\hline
\end{tabular}

TABLE 6 Evaluation of co-authors network influence analysis of one node (Co- SA is Co-Stable Authors, Co- RA is Co- Declining Authors

\begin{tabular}{l|c|c}
\hline Topic: Data Mining & Topic: Database & Topic: Machine Learning \\
\hline Jiawei Hen : Heikki Mannila & Jiawei Hen :Heikki Mannila & Jiawei Hen : Heikki Mannila \\
\hline David Clutter : Arianna Gallo & David Clutter : Heikki Lokki & David Clutter : Heikki Lokki \\
\hline Hasan M. Jamil : Heikki Lokki & Chinying Chaou :Vesa Ollikainen & Hasan M. Jamil: Vesa Ollikainen \\
\hline Larry Travis : Paivi Onkamo & Hasan M. Jamil : Arianna Gallo & Chinying Chaou :Marko Salmenkivi \\
\hline Wo-Shun Luk : Vesa Ollikainen & Wo-Shun Luk :Marko Salmenkivi & Wo-Shun Luk :Arianna Gallo \\
\hline Chinying Chaou : Marko Salmenkivi & Larry Travis :Paivi Onkamo & Larry Travis :Paivi Onkamo \\
\hline
\end{tabular}

with a different professor for his Ph.D. or start working in top research labs. For instance, if you have started with your teaching career which has an almost high workload and less time for research so definitely you didn't find the same motivation or environment to do the research. Below in Table. 7 we declared 30 predicting rising stars(Using citation-based, Paper-based, Author influence based, Specific topic influence based). The best of our information is 
TABLE 7 Predicting rising stars ( $A S$ represent Authors score)

\begin{tabular}{|c|c|c|c|}
\hline & Authors Name & Influence Score & $\mathrm{AS}$ \\
\hline 1 & Wei Ying Ma & $\begin{array}{l}\text { Assistant Managing, } \\
\text { Director Microsoft Research Asia }\end{array}$ & 2.21 \\
\hline 2 & Steffen Staab & $\begin{array}{l}\text { Professor Faculty of Computer Science of the } \\
\text { University of Koblenz Landau }\end{array}$ & 2.20 \\
\hline 3 & Ravi kumar & Senior Staff Research Scientist Google & 2.18 \\
\hline 4 & Jian Pei & $\begin{array}{l}\text { Professor, School of Computing Science, } \\
\text { Simon Fraser University }\end{array}$ & 2.17 \\
\hline 5 & Ian Horrocks & $\begin{array}{l}\text { Professor, Department of Computer Science, } \\
\text { Oxford University }\end{array}$ & 2.15 \\
\hline 6 & P.Jonathon Phillips & $\begin{array}{l}\text { The National Institute of Standards } \\
\text { and Technology, USA }\end{array}$ & 2.14 \\
\hline 7 & Amit Sahai & $\begin{array}{l}\text { Professor, Department of Computer Science, } \\
\text { UCLA, Los Angeles }\end{array}$ & 2.12 \\
\hline 8 & Boualem Benatallah & $\begin{array}{l}\text { Professor, School of Computer Science } \\
\text { and Engineering, New South Wales }\end{array}$ & 2.11 \\
\hline 9 & Berthier Ribeiro Neto & $\begin{array}{l}\text { Associate Professor, Dept. of } \\
\text { CS Federal University of Minas, Gerais }\end{array}$ & 2.10 \\
\hline 10 & Moses Charikar & $\begin{array}{l}\text { Professor, Department of Computer Science, } \\
\text { Princeton University }\end{array}$ & 2.09 \\
\hline 11 & Ling Liu & $\begin{array}{l}\text { Professor, College of Computing, } \\
\text { Georgia Institute of Technology }\end{array}$ & 2.07 \\
\hline 12 & Erik D Demanine & $\begin{array}{l}\text { Professor, MIT Computer Science and } \\
\text { Artificial Intelligence Laboratory, USA }\end{array}$ & 2.06 \\
\hline 13 & Roger Wattenhofer & $\begin{array}{l}\text { Professor, Information Technology } \\
\text { and Electrical Engineering,Switzerland }\end{array}$ & 2.03 \\
\hline 14 & David Blaauw & $\begin{array}{l}\text { Professor, Dept. of Computer Engineering } \\
\text { University of Michigan, Ann Arbor, MI }\end{array}$ & 1.98 \\
\hline 15 & Wei Wang & Professor, Intelligence Control Research Institute,China & 1.95 \\
\hline 16 & venkatesan Guruswami & $\begin{array}{l}\text { Professor, Department of Computer Science, } \\
\text { Carnegie Mellon University }\end{array}$ & 1.93 \\
\hline 17 & Jian Zhang & $\begin{array}{l}\text { Researcher, Institute of Genetics and } \\
\text { Developmental Biology, CAS }\end{array}$ & 1.92 \\
\hline 18 & Marlon Dumas & Professor, University of Tartu & 1.90 \\
\hline 19 & Dennis Sylvester & University of Michigan, Ann Arbor, MI & 1.87 \\
\hline 20 & James T. Kwok & $\begin{array}{l}\text { Professor, Dept. of CS, The Hong Kong } \\
\text { University of Science and Technology }\end{array}$ & 1.86 \\
\hline 21 & Lawrence T. Pileggi & Carnegie Mellon University, Pittsburgh, PA & 1.83 \\
\hline 22 & Chandra Chekuri & $\begin{array}{l}\text { Professor, Department of Computer Science, } \\
\text { University of Illinois }\end{array}$ & 1.81 \\
\hline 23 & Rupak Majumdar & $\begin{array}{l}\text { Professor, Department of Computer Science, } \\
\text { University of California }\end{array}$ & 1.77 \\
\hline 24 & Igor L. Markov & Professor, University of Michigan & 1.72 \\
\hline 25 & Wagner Meira Jr & Professor, Universidade Federal de Minas Gerais & 1.71 \\
\hline 26 & Xin Li & $\begin{array}{l}\text { National Outstanding Youth winner Cold } \\
\text { and Arid Regions Environmental, China }\end{array}$ & 1.24 \\
\hline 27 & Stephen G. Kobourov & $\begin{array}{l}\text { Professor, Department of CS } \\
\text { University of Arizona }\end{array}$ & 1.69 \\
\hline 28 & Orit Hazzan & $\begin{array}{l}\text { Professor, Department of Education in Science, } \\
\text { Israel Institute of Technology }\end{array}$ & 1.24 \\
\hline 29 & Moshe Lewenstein & Bar Ilan University & 1.68 \\
\hline 30 & Masayuki Takeda & Professor, Department of Informatics, Kyushu University & 1.65 \\
\hline
\end{tabular}


TABLE 8 Evaluation of co-authors network influence analysis of one node (Co- SA is Co-Stable Authors, Co- RA is Co- Declining Authors

\begin{tabular}{|c|c|c|c|c|}
\hline Node & Co-Well Established Node & Co-SA Node & Co-DA Node & Co-RA Node \\
\hline David J. Lipman & $\begin{array}{c}\text { John J. Rossi } \\
\text { Temple F. Smith } \\
\text { W. John Wilbur } \\
\text { Michael S. Waterman } \\
\text { Richard W. Pastor } \\
\text { William R. Pearson } \\
\text { Stephen F. Altschul } \\
\text { Raymond J. Carroll } \\
\text { Warren Gish } \\
\text { Webb Miller } \\
\text { James Ostell } \\
\text { Ilene Karsch-Mizrachi } \\
\text { Gregory D. Schuler } \\
\text { Eric W. Sayers } \\
\text { Alejandro A. Sch?ffer } \\
\text { Thomas L. Madden } \\
\text { Eugene V. Koonin } \\
\text { Roman L. Tatusov } \\
\text { J. Zhang } \\
\text { B. F. Francis Ouellette } \\
\text { David L. Wheeler }\end{array}$ & $\begin{array}{c}\text { Barbara A. Rapp } \\
\text { Zheng Zhang }\end{array}$ & $\begin{array}{c}\text { Jacob Maizel } \\
\text { Michael Swatemant } \\
\text { B. Lee } \\
\text { Eugene W. Myers } \\
\text { Dennis Benson } \\
\text { Mark Boguski } \\
\text { D. A. Benson } \\
\text { Karen Clark } \\
\text { Wilma Ross } \\
\text { Arthur Landy } \\
\text { A. A. Schaffer } \\
\text { Jinghui Zhang }\end{array}$ & \\
\hline
\end{tabular}

our first exertion to persuade transfer learning knowledge across different networks to predict and identify the rising stars, well established, stable, declining stars, and influence topic discovery in academic social networks.

In this section, we evaluate the efficiency of $A C T L$ and outcome of expert finding exposed in Table 5, 6, 7, 8 . We perceived that the topic-based social influences, node-based social influences approach can improve the accuracy of expert finding which approved the effectiveness of the ACTL method for topic-base influence analysis and nodebased social influences e. g (David J. Lipman is not predicting rising stars, as well as David J. Lipman, proclaimed predicting rising stars in the previous method. David J. Lipman is well established authors according to definition Tsatsaronis et al. 62 , further node-based social influences approach NICPS discovered 21 co-well established nodes, 2 co-stable, 12 co- declining nodes and 0 predicting stars between the time span from 1995 to 2000 shown in Table 8

We declared predicting rising star between the 0.5 to 1.5 threshold values, shown in Figure. 4 and predicting star accuracy with existing methods. Even though for expert search the different classification algorithms, information retrieval, and graph clustering work were already done $\frac{16|13| 63|64| 65 \mid 66}{}$; however, the aim of this study is to predict upcoming rising stars by means of transfer learning in the area of two or more co-author networks. As far as our knowledge is concerned, no previous work has been done regarding the measuring of topic-level and node-level social influence on large-scale networks classification based on transfer learning and predicting upcoming rising stars.

\section{5 | CONCLUSIONS}

In this area there is a small amount of work is done to predict the rising stars. Its important challenge is to mine the novel scholars that will be a star in the future. We have persuaded a novel approach, Author Classification algorithm using Transfer Learning ( $A C T L$ ) to learn new tasks on target area to mine external knowledge from the source domain. As far as our knowledge is concerned, no previous work has been done regarding the topic-level and node-level social 
influence on large-scale networks to classify using transfer learning to predict and differentiate the well-established, stable and declining authors. The main idea if a junior researcher works with expert researchers having a chance to be experts in the future. In node-weighting schemes, we have designed a node weighting techniques to compute mutual influence scores in specific topic domain, corresponding author mutual influence, co-authorąŕs citations based mutual influence, co-authorąiŕs venues based mutual influence, and co-authorąís paper-based mutual influence schemes with respect to fair contribution. In predicting rising stars schemes, we have calculated Corresponding Author Mutual Influence based on Predicting Stars (CAMIPS), Node-based Influence score Predicting Stars finding(NICPS), and Specific Topic Domain-based Predicting Stars to detect well-established, stable, declining authors, predicting star (STDPS), and topic-based node discovering. We observed that proposed methods highly improved the accuracy of future rising star prediction and achieved superior performances compared with other state-of-art techniques.

\section{Acknowledgement}

This work is partially supported by the National High Technology Research and Development program of China (863 program) under Grant 2014AA012204, the NSFC under Grant 61671018. , Chinese Government Scholarship (CSC),

Competing interests

The authors declare that they have no competing interests.

\section{References}

1. Daud A, Abbasi R, Muhammad F. Finding rising stars in social networks $[\mathrm{C}] / /$ International Conference on Database Systems for Advanced Applications. Springer, Berlin, Heidelberg, 2013: 13-24.

2. Ahmad H A, Wang H. An effective weighted rule-based method for entity resolution[J]. Distributed and Parallel Databases, 2018, 36(3): 593-612.

3. Daud A. Using time topic modeling for semantics-based dynamic research interest finding $[\mathrm{J}]$. Knowledge-Based Systems, 2012, 26: 154-163.

4. Abbasi R, Rehman G, Lee J, et al. Discovering temporal user interest on twitter by using semantic based dynamic interest finding model (TUT) $[\mathrm{C}] / /$ Technology, Networking, Electronic and Automation Control Conference (ITNEC), 2017 IEEE 2nd Information. IEEE, 2017: 743-747.

5. Riaz F M, Abbasi R, Mahmood Z. Adding Temporal Dimension in Social Network by Using Link Analysis[C]//Frontiers of Information Technology (FIT), 2017 International Conference on. IEEE, 2017: 223-228.

6. Daud A, Shaikh A H M A R, Rajpar A H. Scientific reference mining using semantic information through topic modeling[J]. Research Journal of Engineering and Technology, 2009, 28(2): 253-262.

7. Li X L, Foo C S, Tew K L, et al. Searching for rising stars in bibliography networks[C]//International Conference on Database Systems for Advanced Applications. Springer, Berlin, Heidelberg, 2009: 288-292.

8. Daud A, Ahmad M, Malik M S I, et al. Using machine learning techniques for rising star prediction in co-author network[J]. Scientometrics, 2015, 102(2): 1687-1711.

9. Tang J, Zhang J, Yao L, et al. Arnetminer: extraction and mining of academic social networks $[\mathrm{C}] / /$ Proceedings of the 14th ACM SIGKDD international conference on Knowledge discovery and data mining. ACM, 2008: 990-998.

10. Daud A, Li J, Zhou L, et al. Temporal expert finding through generalized time topic modeling[J]. KnowledgeBased Systems, 2010, 23(6): 615-625.

11. Li S, Sun Y, Soergel D. Automatic Decision Support for Clinical Diagnostic Literature Using Link Analysis in a Weighted Keyword Network[J]. Journal of medical systems, 2018, 42(2): 27.

12. Kim J. Evaluating author name disambiguation for digital libraries: a case of DBLP[J]. Scientometrics, 2018, 116(3): 1867-1886. 
13. Santos R L T, Macdonald C, Ounis I. Learning to rank query suggestions for adhoc and diversity $\operatorname{search}[\mathrm{J}]$. Information Retrieval, 2013, 16(4): 429-451.

14. Amjad T, Daud A, Aljohani N R. Ranking authors in academic social networks: a survey[J]. Library Hi Tech, 2018.

15. Nerurkar P, Pavate A, Shah M, et al. Analysis of Probabilistic Models for Influence Ranking in Social Networks[M]//Computing, Communication and Signal Processing. Springer, Singapore, 2019: 215-223.

16. Wang G A, Jiao J, Abrahams A S, et al. ExpertRank: A topic-aware expert finding algorithm for online knowledge communities[J]. Decision Support Systems, 2013, 54(3): 1442-1451.

17. Boldi P, Santini M, Vigna S. PageRank as a function of the damping factor[C]//Proceedings of the 14th international conference on World Wide Web. ACM, 2005: 557-566.

18. Mallick C, Das A K, Dutta M, et al. Graph-Based Text Summarization Using Modified TextRank[M]//Soft Computing in Data Analytics. Springer, Singapore, 2019: 137-146.

19. Gong X, Lee M K O, Liu Z, et al. Examining the Role of Tie Strength in Usersąr Continuance Intention of Second-Generation Mobile Instant Messaging Services[J]. Information Systems Frontiers, 2018: 1-22.

20. Arul R, Moorthy R S, Bashir A K. Ensemble Learning Mechanisms for Threat Detection: A Survey[M]//Machine Learning and Cognitive Science Applications in Cyber Security. IGI Global, 2019: 240-281.

21. Ali A, Rafique H, Arshad T, et al. A fractal-based authentication technique using sierpinski triangles in smart devices[J]. Sensors, 2019, 19(3): 678.

22. Kutia S, Chauhdary S H, Iwendi C, et al. Socio-Technological factors affecting user adoption of eHealth functionalities: A case study of China and Ukraine eHealth systems[J]. IEEE Access, 2019, 7: 90777-90788.

23. Aggarwal C C, Li N. On node classification in dynamic content-based networks[C]//Proceedings of the 2011 SIAM International Conference on Data Mining. Society for Industrial and Applied Mathematics, 2011: 355-366.

24. He Z F, Yang M, Gao Y, et al. Joint multi-label classification and label correlations with missing labels and feature selection[J]. Knowledge-Based Systems, 2019, 163: 145-158.

25. Pan S J, Yang Q. A survey on transfer learning[J]. IEEE Transactions on knowledge and data engineering, 2010, 22(10): 1345-1359.

26. Song A, Liu Y, Wu Z, et al. A local random walk model for complex networks based on discriminative feature combinations[J]. Expert Systems with Applications, 2019, 118: 329-339.

27. Bhagat S, Cormode G, Muthukrishnan S. Node classification in social networks $[\mathrm{M}] / /$ Social network data analytics. Springer US, 2011: 115-148.

28. Zhang Y, Xiong Y, Kong X, et al. Deep Collective Classification in Heterogeneous Information Networks $[\mathrm{C}] / /$ Proceedings of the 2018 World Wide Web Conference on World Wide Web. International World Wide Web Conferences Steering Committee, 2018: 399-408.

29. Gupta S, Khattar A, Gogia A, et al. Collective Classification of Spam Campaigners on Twitter: A Hierarchical Meta-Path Based Approach[J]. arXiv preprint arXiv:1802.04168, 2018.

30. Eldardiry H, Neville J. Across-Model Collective Ensemble Classification[C]//AAAI. 2011.

31. Sudharshan P J, Petitjean C, Spanhol F, et al. Multiple instance learning for histopathological breast cancer image classification[J]. Expert Systems with Applications, 2019, 117: 103-111.

32. Kalra V, Agrawal R. Challenges of Text Analytics in Opinion Mining[M]//Extracting Knowledge From Opinion Mining. IGI Global, 2019: 268-282. 
33. Witten I H, Frank E, Hall M A, et al. Data Mining: Practical machine learning tools and techniques[M]. Morgan Kaufmann, 2016.

34. Dai W, Yang Q, Xue G R, et al. Boosting for transfer learning $[\mathrm{C}] / /$ Proceedings of the 24th international conference on Machine learning. ACM, 2007: 193-200.

35. Plekhanova E, Nuzhdin S V, Utkin L V, et al. Prediction of deleterious mutations in coding regions of mammals with transfer learning[J]. Evolutionary Applications, 2019, 12(1): 18-28.

36. Cao W, Qian S, Wu S, et al. Unsupervised Multi-task Learning with Hierarchical Data Structure[J]. Pattern Recognition, 2019, 86: 248-264.

37. Jing B, Lu C, Wang D, et al. Cross-Domain Labeled LDA for Cross-Domain Text Classification[C]//2018 IEEE International Conference on Data Mining (ICDM). IEEE, 2018: 187-196.

38. Li Z, Wei Y, Zhang Y, et al. Hierarchical Attention Transfer Network for Cross-domain Sentiment Classification $[\mathrm{C}] / /$ Proceedings of the Thirty-Second AAAI Conference on Artificial Intelligence, AAAI 2018, New Orleans, Lousiana, USA, February 2íC7, 2018. 2018.

39. Cordella L P, Foggia P, Sansone C, et al. An improved algorithm for matching large graphs[C]//3rd IAPR-TC15 workshop on graph-based representations in pattern recognition. 2001: 149-159.

40. Jensen D, Neville J, Gallagher B. Why collective inference improves relational classification $[\mathrm{C}] / /$ Proceedings of the tenth ACM SIGKDD international conference on Knowledge discovery and data mining. ACM, 2004: 593-598.

41. Neville J, Jensen D. Iterative classification in relational data[C]//Proc. AAAI-2000 Workshop on Learning Statistical Models from Relational Data. 2000: 13-20.

42. Bilgic M, Namata G M, Getoor L. Combining collective classification and link prediction[C]//Seventh IEEE International Conference on Data Mining Workshops (ICDMW 2007). IEEE, 2007: 381-386.

43. Liang W, Xie H, Rao Y, et al. Universal affective model for Readers' emotion classification over short texts[J]. Expert Systems with Applications, 2018, 114: 322-333.

44. Rao Y, Xie H, Li J, et al. Social emotion classification of short text via topic level maximum entropy model[J]. Information and Management, 2016, 53(8): 978-986.

45. Lu Y, Dong R, Smyth B. Coevolutionary Recommendation Model: Mutual Learning between Ratings and Reviews $[\mathrm{C}] / /$ Proceedings of the 2018 World Wide Web Conference on World Wide Web. International World Wide Web Conferences Steering Committee, 2018: 773-782.

46. Jiang L, Zhao X, Ge B, et al. An efficient algorithm for mining a set of influential spreaders in complex networks[J]. Physica A: Statistical Mechanics and its Applications, 2019, 516: 58-65.

47. Granovetter M S. The strength of weak ties[M]//Social networks. 1977: 347-367.

48. Valenzuela S, Correa T, Gil de Zĺš?iga H. Ties, likes, and tweets: Using strong and weak ties to explain differences in protest participation across Facebook and Twitter use[J]. Political Communication, 2018, 35(1): 117-134.

49. Rane R. Frequent Subpatterns Distribution in Social Network Analysis[M]//Emerging Technologies in Data Mining and Information Security. Springer, Singapore, 2019: 393-403.

50. Chaturvedi S, Saritha S K. Parallel Frequent Pattern Mining on Natural Language Based Social Media Data[M]//Emerging Technologies in Data Mining and Information Security. Springer, Singapore, 2019: 507-517.

51. Shi C, Hu B, Zhao X,. Heterogeneous Information Network Embedding for Recommendation[J]. IEEE Transactions on Knowledge and Data Engineering, 2018. 
52. Kschischang F R, Frey B J, Loeliger H A. Factor graphs and the sum product algorithm[J]. IEEE Transactions on information theory, 2001, 47(2): 498-519.

53. Treur J. The ins and outs of network-oriented modeling: from biological networks and mental networks to social networks and beyond[M]//Transactions on Computational Collective Intelligence $X X X I I$. Springer, Berlin, Heidelberg, 2019: 120-139.

54. Tang J, Lou T, Kleinberg J. Transfer learning to infer social ties across heterogeneous networks $[\mathrm{J}]$. ACM Transactions on Information Systems (TOIS), 2016, 34(2): 7.

55. Rehman G, Lee J, Abbasi R, et al. Quantifying the Impact of Hot-paper on new Researchers[C]//Proceedings of the 2017 2nd International Conference on Communication and Information Systems. ACM, 2017: 329-334.

56. DBLP bibliography database, http : //www.informatik.uni-trier.de/ley/db/

57. CiteSeer.IST scientific literature digital library. [http: //citeseer.ist.psu.edu].

58. McCallum A K, Nigam K, Rennie J. Automating the construction of internet portals with machine learning[J]. Information Retrieval, 2000, 3(2): 127-163.

59. Sen P, Namata G, Bilgic M. Collective classification in network data[J]. AI magazine, 2008, 29(3): 93.

60. McDowell L K, Gupta K M, Aha D W. Cautious collective classification[J]. Journal of Machine Learning Research, 2009, 10(Dec): 2777-2836.

61. Fang M, Yin J, Zhu X. Transfer learning across networks for collective classification[C]//Data Mining (ICDM), 2013 IEEE 13th International Conference on. IEEE, 2013: 161-170.

62. Tsatsaronis G, Varlamis I, Torge S. How to become a group leader or modeling author types based on graph mining[C]//International Conference on Theory and Practice of Digital Libraries. Springer, Berlin, Heidelberg, 2011: 15-26.

63. Bu Z, Gao G, Li H J, et al. CAMAS: A cluster-aware multiagent system for attributed graph clustering[J]. Information Fusion, 2017, 37: 10-21.

64. Bu Z, Li H J, Cao J. Dynamic Cluster Formation Game for Attributed Graph Clustering[J]. IEEE Transactions on Cybernetics, 2017 (99): 1-14.

65. Xiong Z, Xia C, Sun B. Mining Similarity of Users in Location-Based Social Networks for Discovering Overlapping Communities $[\mathrm{M}] / /$ Recent Developments in Intelligent Computing, Communication and Devices. Springer, Singapore, 2019: 417-428.

66. Feng L, Ali A, Iqbal M, et al. Optimal Haptic Communications over Nanonetworks for E-Health Systems[J]. IEEE Transactions on Industrial Informatics, 2019, 15(5): 3016-3027. 